\title{
Numerical integration of polynomials and discontinuous functions on irregular convex polygons and polyhedrons
}

\author{
S. E. Mousavi · N. Sukumar
}

Received: 5 October 2010 / Accepted: 26 November 2010 / Published online: 16 December 2010

(C) The Author(s) 2010. This article is published with open access at Springerlink.com

\begin{abstract}
We construct efficient quadratures for the integration of polynomials over irregular convex polygons and polyhedrons based on moment fitting equations. The quadrature construction scheme involves the integration of monomial basis functions, which is performed using homogeneous quadratures with minimal number of integration points, and the solution of a small linear system of equations. The construction of homogeneous quadratures is based on Lasserre's method for the integration of homogeneous functions over convex polytopes. We also construct quadratures for the integration of discontinuous functions without the need to partition the domain into triangles or tetrahedrons. Several examples in two and three dimensions are presented that demonstrate the accuracy and versatility of the proposed method.
\end{abstract}

Keywords Numerical integration - Lasserre's method · Euler's homogeneous function theorem .

Irregular polygons and polyhedrons $\cdot$ Homogeneous and nonhomogeneous functions - Strong and weak discontinuities · Polygonal finite elements .

Extended finite element method

\section{Introduction}

Numerical integration is an important ingredient within many solution techniques in engineering and the sciences. With the development of new numerical methods, the need for

National Science Foundation, CMMI-0626481, DMS-0811025.

S. E. Mousavi · N. Sukumar $(\bowtie)$

Department of Civil and Environmental Engineering,

University of California, Davis, CA 95616, USA

e-mail: nsukumar@ucdavis.edu accurate and efficient integration schemes has emanated, due to both the general shapes of the integration domain and the presence of generalized classes of functions. Conforming polygonal finite elements [1-4] and finite elements on convex polyhedra [5-7] require the integration of nonpolynomial basis functions. The integration of polynomials on irregular polytopes arises in the non-conforming variable-element-topology finite element method [8,9], discontinuous Galerkin finite elements [10], finite volume element method [11] and mimetic finite difference schemes [12-14]. In partition-of-unity methods such as the extended finite element method (X-FEM) [15,16], discontinuous functions are integrated to form the stiffness matrix of elements that are cut by a crack or an interface. Versatility of such methods is due to their power to handle more complicated internal geometries, albeit at the expense of greater demands on numerical integration, which points to the need for devising flexible and efficient numerical integration techniques.

In this paper, we focus on the integration of polynomials on irregular convex polygons and polyhedra. In two dimensions, the boundaries of the integration region are contained in straight lines and in three dimensions, the domain is bounded by planes. Also, we are particularly interested in cases where the integrand is not known explicitly, but can be evaluated at any point within the domain of integration. Such cases arise in finite-element and partition-of-unity methods. There are few choices for the integration of polynomials over general two- and three-dimensional regions. Most of the available methods in the literature are appropriate for special forms of polynomials [17-19], or are restricted to the integration on simplexes and regular polytopes [20-22]. The most frequently used strategy for the integration of a polynomial over an irregular polygon or polyhedron is to decompose it into triangles or tetrahedrons and apply well-known integration rules to each partition. Integration of polynomials 
over $n$-dimensional polyhedra can also be done by lowerdimensional integrations over the boundary of the domain using divergence theorem and/or Green's theorem [23-28], or using Euler's theorem in case of homogeneous functions [17,18]. A method is presented in Ref. [19] for the efficient integration of powers of linear forms over simplexes in very high dimensions.

In this paper, we use moment fitting equations to construct quadrature rules for the integration of polynomials on irregular convex polygons and polyhedra. The moment fitting technique is independent of the shape of the domain or type of the basis functions, and enables one to construct integration rules with desirable properties, such as interiority of points and symmetry. These properties are enforced through adding appropriate constraints to the moment equations. In Ref. [29], moment fitting was used together with the node elimination algorithm [30], to construct and optimize quadratures for the integration of polynomials over arbitrary polygons. The moment equations contain the integration of the basis functions (polynomials of total degree up to $d$ ) over the domain. These integrations were performed algebraically in Ref. [29]. Although algebraic integration of polynomials is straightforward in two dimensions, it is not generally a well-posed problem in three dimensions, and therefore extending the technique of Ref. [29] to three dimensions is unwieldy. It bears mention that for monomials one can use divergence theorem to transform the surface integrals to line integrals [8], which can be handled efficiently. In three dimensions, successive application of the divergence theorem results in line integrals over the edges of the polyhedron $[9,26,28]$. Lasserre's method $[17,18]$, which is a technique for the integration of homogeneous functions on convex polygons and polyhedra based on Euler's theorem, transforms the integration of a homogeneous function on a convex polygon to line integrations over its edges. Similarly, one can apply the technique twice for the integration over a convex polyhedron and transform the volume integrals into line integrals. Lasserre's formula has advantages over the divergence theorem: calculation of the normals to the boundary and projections on the plane are not required and the integrand remains unchanged. The basis functions of the moment fitting equations are monomials with respect to the spatial coordinates, which makes Lasserre's method ideal for their integration.

The structure of this paper is as follows. Lasserre's method for the integration of homogeneous functions is explained and the main theorems are presented in Sect. 2.1. A method for constructing quadratures for the integration of homogeneous functions based on Lasserre's method is presented in Sect. 2.2. These homogeneous quadratures are used throughout the paper for the integration of the basis functions. In Sect. 2.3, we propose a technique for the integration of a linear combination of homogeneous functions, when the func- tion is not known explicitly, based on Lasserre's method and the solution of a small system of linear equations. We describe the algorithm for the construction of quadratures for irregular convex polygons in Sect. 3, followed by several examples. In Sect. 4, we consider the integration of functions with strong and weak discontinuities and demonstrate the applicability of the method through a few examples. We extend the quadrature construction scheme to three dimensions in Sect. 5, both for polynomial functions and discontinuous functions. We integrate a cubic polynomial on a convex polyhedron and present two practical examples that arise in crack-modeling using the X-FEM. The main findings of this study are summarized and some concluding remarks are made in Sect. 6.

\section{Lasserre's method for integration of homogeneous functions}

\subsection{Lasserre's method}

Lasserre presented a method for the integration of positively homogeneous functions over convex polytopes $[17,18]$. Integration in $\Omega \subset \mathbb{R}^{n}$ is reduced to a number of weighted integrations over the $(n-1)$-dimensional faces of $\Omega$, i.e., $\Omega_{i} \subset$ $\mathbb{R}^{n-1}$ for $i=1,2, \ldots, m$, through the application of Euler's theorem. The weights of the integrations are functions of the geometry of $\Omega$ and the degree of homogeneity. The integrations in $(n-1)$ dimensions can be done using standard integration schemes in an efficient way. Under certain circumstances the reduction of the integrations to even lower dimensions is also possible.

Let $f: \mathbb{R}^{n} \rightarrow \mathbb{R}$ be a real continuous positively homogeneous function of degree $q, f(\lambda \mathbf{x})=\lambda^{q} f(\mathbf{x})$ for all $\lambda>0$ and $\mathbf{x} \in \mathbb{R}^{n}$. Moreover, assume that the domain of integration $\Omega \subset \mathbb{R}^{n}$ is a convex polytope, which is described as $\{\mathbf{x} \in$ $\left.\mathbb{R}^{n}: \mathbf{A x} \leq \mathbf{b}\right\}$, with $\mathbf{A}$ a real $m \times n$ matrix and $\mathbf{b}$ a real vector of length $m$. Also, let $\Omega_{i}$ be the $(n-1)$-dimensional face of $\Omega: \Omega_{i}=\left\{\mathbf{x} \in \mathbb{R}^{n}: \mathbf{A x} \leq b, \mathbf{A}_{i} \mathbf{x}=b_{i}\right\}$ is determined by the hyperplane $\mathbf{A}_{i} \mathbf{x}=b_{i}$ with $\mathbf{A}_{i}$ being the $i$ th row of $\mathbf{A}$. The face $\Omega_{i}$ is contained in the $(n-1)$-dimensional variety ${ }^{1} \mathcal{H}_{i}$ and the algebraic distance from the point $\mathbf{x}_{0}$ to $\mathcal{H}_{i}$ is denoted by $d\left(\mathbf{x}_{0}, \mathcal{H}_{i}\right)$ and can be calculated as $d\left(\mathbf{x}_{0}, \mathcal{H}_{i}\right)=$ $\left(b_{i}-\mathbf{A}_{i} \mathbf{x}_{0}\right) /\left\|\mathbf{A}_{i}\right\|$, with $\|\cdot\|$ denoting the Euclidean norm. The following theorems from Ref. [17] describe the method. These theorems are based on Euler's homogeneous function theorem, namely

$q f(\mathbf{x})=\langle\nabla f(\mathbf{x}), \mathbf{x}\rangle \quad \forall \mathbf{x}$,

where $f$ is a continuously differentiable function and $\langle\cdot, \cdot\rangle$ denotes the inner product of vectors.

\footnotetext{
1 A variety is the extension of the algebraic curves to higher dimensions, or more precisely, a set of points that satisfy a system of polynomial equations.
} 
Remark Throughout this paper, we will interchangeably use the tensor representation of coordinates $\left\{x_{i}\right\}_{i=1}^{3}$, and the Cartesian representation $\{x, y, z\}$.

Theorem 1 (Integration of homogeneous functions) Assume that $f$ is continuously differentiable, $\mathcal{V}_{n}(\Omega) \neq 0$, and for all $i=1, \ldots, m, \mathcal{V}_{n-1}\left(\Omega_{i}\right) \neq 0$. Then

$$
\begin{aligned}
\int_{\Omega} f(\mathbf{x}) d \mathbf{x} & =\frac{1}{n+q} \sum_{i=1}^{m} \frac{b_{i}}{\left\|\mathbf{A}_{i}\right\|} \int_{\Omega_{i}} f d \mu \\
& =\sum_{i=1}^{m} \frac{d\left(\mathbf{o}, \mathcal{H}_{i}\right)}{n+q} \int_{\Omega_{i}} f d \mu,
\end{aligned}
$$

where $d \mu$ is the Lebesgue measure on the $(n-1)$-dimensional affine variety $\mathcal{H}_{i}$ that contains $\Omega_{i}\left(d \mu=\left\|\mathbf{A}_{i}\right\| d x\right)$ and $\mathbf{0}$ is the origin. $\mathcal{V}_{n}$ and $\mathcal{V}_{n-1}$ denote the $n$ - and $(n-1)$-dimensional volume, respectively.

Theorem 2 (Further reduction of integration of homogeneous functions) Let $f$ be twice continuously differentiable and for all $i=1, \ldots, m$, either $\Omega_{i}=\emptyset$ or $\mathcal{V}_{n-1}\left(\Omega_{i}\right) \neq 0$. Also, assume that for every $j=1, \ldots, m, j \neq i$, either $\Omega_{i j}=\emptyset$ or $\mathcal{V}_{n-2}\left(\Omega_{i j}\right) \neq 0$. Then

$$
\begin{aligned}
\int_{\Omega_{i}} f d \mu=\frac{1}{n+q-1} & {\left[\sum_{j \neq i} d_{i}\left(\mathbf{x}_{0}, \mathcal{H}_{i j}\right) \int_{\Omega_{i j}} f d v\right.} \\
& \left.+\int_{\Omega_{i}}\left\langle\nabla f, \mathbf{x}_{0}\right\rangle d \mu\right],
\end{aligned}
$$

where $\Omega_{i j}$ is the $(n-2)$-dimensional face of $\Omega$ defined as $\Omega_{i j}=\left\{\mathbf{x} \in \Omega, \mathbf{A}_{i} \mathbf{x}=b_{i}, \mathbf{A}_{j} \mathbf{x}=b_{j}\right\}$, and $\mathcal{H}_{i j}$ is the affine variety that contains $\Omega_{i j}$. For example, in two dimensions with $\Omega$ being a polygon, $\Omega_{i}$ is the ith edge and $\Omega_{i j}$ is the vertex of the polygon connecting edges $i$ and $j$. In three dimensions, $\Omega_{i}$ is the ith face of the polytope and $\Omega_{i j}$ is the line segment at the intersection of faces $i$ and $j . d v$ is the Lebesgue measure on the $(n-2)$-dimensional affine variety $\mathcal{H}_{i j} \subset \mathcal{H}_{i}$, and $\mathbf{x}_{0} \in \mathcal{H}_{i}$ is a fixed point that can be selected arbitrarily.

In Sect. 2.2, we construct quadrature rules based on Theorem 1 with the quadrature points on the faces of the integration region. The resulting quadratures are suitable for the integration of homogeneous functions. In two dimensions, the faces of the region are line segments. Hence, these quadratures reduce the area integration to line integrations. The integration can be further reduced to function evaluation at polygonal vertices using Theorem 2 . However, this is avoided since optimal one-dimensional Gauss quadrature rules are available. In three dimensions, we use the above theorems to reduce the volume integrals to line integrals and then apply one-dimensional Gauss quadrature rules.

\subsection{Quadratures for homogeneous functions}

Theorem 1 can be used to construct quadrature rules for the integration of homogeneous functions over the integration region $\Omega$ with all the integration points located on the faces of the region $\Omega_{i}$. Assume that $\overline{\mathcal{Q}}_{i}$ is a $n s p_{i}$-point quadrature over $\Omega_{i}$ with the integration points and weights $\left\{\overline{\mathbf{x}}_{i}^{a}, \bar{w}_{i}^{a}\right\}_{a=1}^{n s p_{i}}$ and define the operation of $\overline{\mathcal{Q}}_{i}$ on a function $f$ as:

$$
\overline{\mathcal{Q}}_{i}(f)=\sum_{a=1}^{n s p_{i}} \bar{w}_{i}^{a} f\left(\overline{\mathbf{x}}_{i}^{a}\right) \approx \int_{\Omega_{i}} f d \mu .
$$

Now, obtain the modified quadrature $\mathcal{Q}_{i}$ with points and weights $\left\{\mathbf{x}_{i}^{a}, w_{i}^{a}\right\}_{a=1}^{n s p_{i}}$ using:

$\mathbf{x}_{i}^{a}=\overline{\mathbf{x}}_{i}^{a}, \quad w_{i}^{a}=d\left(\mathbf{o}, \mathcal{H}_{i}\right) \bar{w}_{i}^{a}$.

The combination of the quadrature rules $\mathcal{Q}=\left\{\mathcal{Q}_{i}\right\}_{i=1}^{m}$ constructed over the $m$ faces of $\Omega$, is a quadrature, with $n s p=$ $\sum_{i=1}^{m} n s p_{i}$ integration points on the faces of the domain, that can integrate $q$-homogeneous functions over the integration region:

$$
\mathcal{Q}(f) \approx(n+q) \int_{\Omega} f(\mathbf{x}) d \mathbf{x} .
$$

We will refer to such quadratures as homogeneous quadratures throughout this paper, since they can only be used to integrate homogeneous functions. For example, in a twodimensional setting, with $\Omega$ being a convex $m$-gon and $\Omega_{i}$ denoting the $i$ th edge of the polygon, one can obtain $\overline{\mathcal{Q}}_{i}-\mathrm{a}$ standard Gauss quadrature rule over the interval $[-1,1]$ is mapped to the line-segment $\Omega_{i}$ and the weights of the quadrature are multiplied by the length of the $i$ th edge of the polygon divided by two.

The approximation sign in (4) and (6) pertains to the approximation error of the quadratures $\overline{\mathcal{Q}}_{i}$, and no further approximation is introduced in the construction. In other words, beginning with quadratures that are exact for the integration of $f$ over the faces of the region, for example a Gauss quadrature rule for polynomial $f$, one can obtain an exact quadrature via (6). The application of homogeneous quadratures is illustrated through the following two examples.

\section{Example 1: Homogeneous quadrature over bi-unit square}

We would like to construct a homogeneous quadrature for the integration of monomials up to order 5 on the bi-unit square $[-1,1]^{2}$. In one dimension, a 3-point Gauss quadrature can integrate polynomials up to order 5 exactly. We take the 3-point Gauss quadrature with the coordinates $\xi=\{-0.774596669241483,0,0.774596669241483\}$ and weights $w=\{0.555555555555556,0.888888888888889$, $0.555555555555556\}$ and map it to the edges of the square. 
Table 1 Positions and weights of the homogeneous quadrature over the bi-unit square a $n=2$ in two dimensions

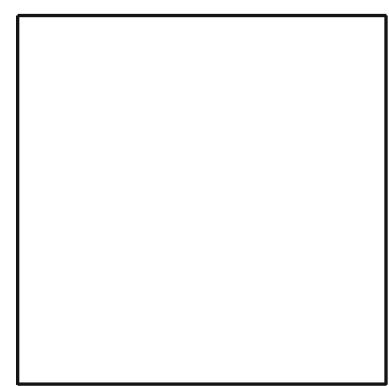

(a)

\begin{tabular}{llll}
\hline & \multicolumn{1}{l}{$y$} & \multicolumn{1}{l}{ Weight } \\
\hline 1 & -0.7745966692414834 & -1.0000000000000000 & 0.55555555555555556 \\
2 & 0.0000000000000000 & -1.0000000000000000 & 0.8888888888888888 \\
3 & 0.7745966692414834 & -1.0000000000000000 & 0.5555555555555556 \\
4 & 1.0000000000000000 & -0.7745966692414834 & 0.55555555555555556 \\
5 & 1.0000000000000000 & 0.0000000000000000 & 0.8888888888888888 \\
6 & 1.0000000000000000 & 0.7745966692414834 & 0.5555555555555556 \\
7 & 0.7745966692414834 & 1.0000000000000000 & 0.5555555555555556 \\
8 & 0.0000000000000000 & 1.0000000000000000 & 0.8888888888888888 \\
9 & -0.7745966692414834 & 1.0000000000000000 & 0.5555555555555556 \\
10 & -1.0000000000000000 & 0.7745966692414834 & 0.5555555555555556 \\
11 & -1.0000000000000000 & 0.0000000000000000 & 0.8888888888888888 \\
12 & -1.0000000000000000 & -0.7745966692414834 & 0.5555555555555556 \\
\hline$f$ & $q$ & $\frac{\mathcal{Q} \square(f)}{n+q}$ & $\left|\int_{\square} f d A-\frac{\mathcal{Q}_{\square}(f)}{n+q}\right|^{\mathrm{a}}$ \\
\hline 1 & 0 & 4 & 0 \\
$x$ & 1 & 0 & 0 \\
$x^{2} y^{2}$ & 4 & 0.444444444444445 & $1.6 \times 10^{-16}$ \\
$x^{2} y^{3}$ & 5 & 0 & 0 \\
\hline
\end{tabular}

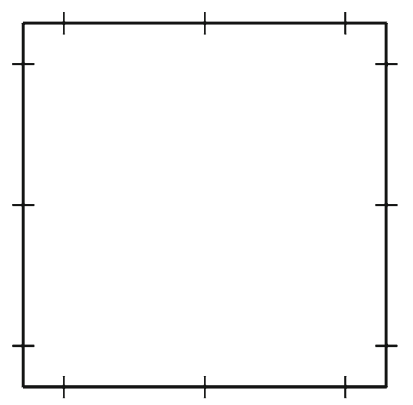

(b)
Fig. 1 Homogeneous quadrature over the bi-unit square. a Bi-unit square; and $\mathbf{b}$ homogeneous quadrature with 12 points

Also, the weights of the quadrature are modified according to (5) (see Table 1 and Fig. 1b for the positions and weights of the quadrature). Let us call this quadrature $\mathcal{Q} \square$. In Table 1, we use $\mathcal{Q} \square$ to integrate a few (homogeneous) bivariate monomials and present the error of integration - as expected, all integrations are exact to within machine precision. We use similar quadratures in Sects. 3 and 4 to integrate the basis functions in our quadrature construction scheme.

\section{Example 2: Homogeneous quadrature over a regular hexagon}

We construct homogeneous quadratures with $n s p$ points over each of the edges of the regular hexagon shown in Fig. 2a. The hexagon is built over a unit circle centered at the origin. A sample quadrature with $n s p=5$ is shown in Fig. 2 b.
The aim is to integrate $f(r)=1 / r^{\alpha}$ over the regular hexagon, with $r$ being the distance from the origin. The function $1 / r^{\alpha}$ is homogeneous with degree of homogeneity $-\alpha$. Since the domain of integration contains the origin, the integrand is weakly singular, and domain integration using standard quadratures over the triangle requires many integration points to obtain the desired accuracy. To calculate the reference solution, we use the generalized Duffy transformation [31] after partitioning the hexagon into 6 triangles by connecting the origin to the vertices of the hexagon. We increase $n s p$ so that the result approaches the reference value-the efficiency of the method for $f(r)=1 / \sqrt{r}$ and $f(r)=1 / r$ is demonstrated in Fig. 2c.

The above technique for finding $\mathcal{Q}$ is straightforward and provides flexibility when the integrand is homogeneous. For example, in the node elimination algorithm [29,30,32], the basis functions can be selected as monomials that are homogeneous functions (see Sect. 3). However, when the integrand is a linear combination of homogeneous functions, each of the terms must be known explicitly so that they can be integrated separately. This limitation renders the homogeneous quadratures impractical for finite-element or boundary-element methods, where the integrands are known implicitly and can only be evaluated at given points. To clarify this issue, let $g(\mathbf{x})=\sum_{j} g_{j}(\mathbf{x})$, with $g_{j}$ being $q_{j}$-homogeneous. The following integral is valid:

$$
\int_{\Omega} g(\mathbf{x}) d \mathbf{x} \approx \sum_{j} \frac{\mathcal{Q}\left(g_{j}\right)}{n+q_{j}},
$$




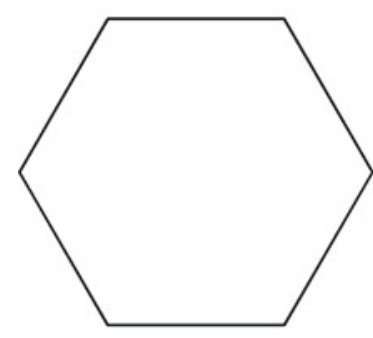

(a)

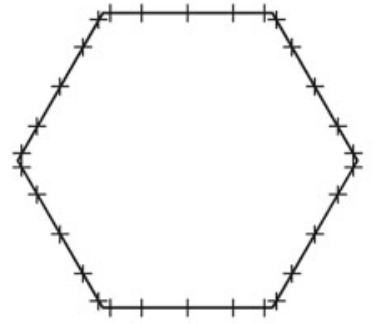

(b)

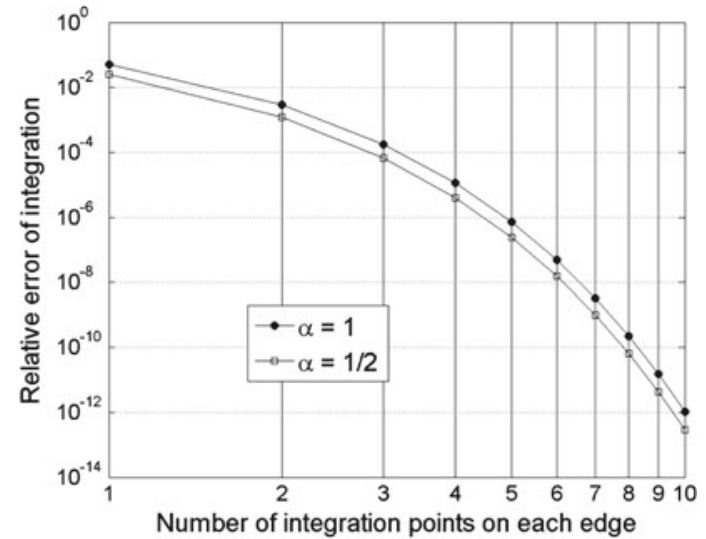

(c)

Fig. 2 Homogeneous quadrature over regular hexagon. a Regular hexagon on a unit circle; b homogeneous quadrature with 30 points; and c relative error of calculating $\int_{\square} 1 / r^{\alpha} d A$ using homogeneous quadratures

where $\mathcal{Q}$ is the quadrature obtained through (5). If each $g_{j}(\mathbf{x})$ is not known explicitly, then the value of the integral in (7) can not be computed. In the next section, a solution is devised for this problem.

\subsection{Application of Lasserre's method for the integration of a linear combination of homogeneous functions}

Lasserre's method of integration is an elegant technique for the integration over convex regions. However, the technique and the corresponding quadrature rules (see Sect. 2.2) are limited to homogeneous functions. In the case of a linear combination of homogeneous functions, additional information is required to apply the method: each of the terms in the sum must be known explicitly and integration is performed for each term individually. In this section, we use the homogeneous quadratures for the integration of a linear combination of homogeneous functions without knowing or evaluating each one of them separately.

Assume that $\mathcal{Q}$ is a homogeneous quadrature that can integrate a class of functions $\Phi$ over the region $\Omega \subset \mathbb{R}^{n}$ using (6). Also assume that $g(\mathbf{x})$ can be written as the sum of $m$ homogeneous functions: $g(\mathbf{x})=\sum_{i=1}^{m} g_{i}(\mathbf{x})$, with $g_{i} \in \Phi$ being $q_{i}$-homogeneous, $g_{i}(\lambda \mathbf{x})=\lambda^{q_{i}} g_{i}(\mathbf{x})$ for all $\lambda \in \mathbb{R}, \lambda>0$. Note that $g_{i}$ can be the summation of a group of $q_{i}$-homogeneous functions (can contain more than one term). We also assume that $g$ is well-defined everywhere in $\mathbb{R}^{n}$ and can be evaluated even outside the region $\Omega$. One can use (7) to integrate $g$ over the domain, provided that $\left\{g_{i}\right\}_{i=1}^{m}$ are known individually. Here we assume that $g$ can only be evaluated at certain points (quadrature points) and the functions $g_{i}$ are not given; nor do we wish to approximate $g$ explicitly with a few homogeneous functions. According to the definition of homogeneity, and by writing the integration of a sum as the sum of integrals, the following is valid:

$$
\int_{\Omega} g(\lambda \mathbf{x}) d \mathbf{x}=\int_{\Omega} \sum_{i=1}^{m} g_{i}(\lambda \mathbf{x})=\sum_{i=1}^{m} \lambda^{q_{i}} \int_{\Omega} g_{i}(\mathbf{x}) d \mathbf{x} .
$$

Also, the operation of $\mathcal{Q}$ on $g$ can be simplified as:

$$
\mathcal{Q}(g)=\mathcal{Q}\left(\sum_{i=1}^{m} g_{i}\right)=\sum_{i=1}^{m} \mathcal{Q}\left(g_{i}\right)=\sum_{i=1}^{m}\left(q_{i}+n\right) \int_{\Omega} g_{i}(\mathbf{x}) d \mathbf{x},
$$

where (6) was used in the derivation of the last term. By exploiting the homogeneity of $g_{i}$, one can write:

$\mathcal{Q}(g(\lambda \mathbf{x}))=\sum_{i=1}^{m} \lambda^{q_{i}}\left(q_{i}+n\right) \int_{\Omega} g_{i}(\mathbf{x}) d \mathbf{x}$.

The term on left-hand-side of (10) can be evaluated by manipulating the positions of the integration points of $\mathcal{Q}$ : $\mathcal{Q}(g(\lambda \mathbf{x}))=\sum_{i=1}^{n s p} w_{i} g\left(\lambda \mathbf{x}_{i}\right)$ where $\left\{\mathbf{x}_{i}, w_{i}\right\}_{i=1}^{n s p}$ are the integration points and weights of $\mathcal{Q}$. Consider a new quadrature $\mathcal{Q}^{\lambda}$ whose points and weights are defined as $\left\{\mathbf{x}_{i}^{\lambda}=\lambda \mathbf{x}_{i}, w_{i}^{\lambda}=\right.$ $\left.w_{i}\right\}_{i=1}^{n s p}$. Then, one can write:

$\mathcal{Q}(g(\lambda \mathbf{x}))=\mathcal{Q}^{\lambda}(g(\mathbf{x}))$.

We arrive at the final equation by substituting (11) into (10) and replacing the integrals $\int_{\Omega} g_{i} d \mathbf{x}$ with $I_{i}$ :

$\mathcal{Q}^{\lambda}(g)=\sum_{i=1}^{m} \lambda^{q_{i}}\left(q_{i}+n\right) I_{i}$.

Equation (12) has $m$ unknowns $\left\{I_{i}\right\}_{i=1}^{m}$, and holds for any given $\lambda>0$. It is straightforward to transform (12) into a linear system of equations by selecting $m$ values for $\lambda$ and evaluating the left-hand-side for each of them, as follows: 


$$
\begin{aligned}
& \left(\begin{array}{c}
\mathcal{Q}^{\lambda_{1}}(g) \\
\mathcal{Q}^{\lambda_{2}}(g) \\
\vdots \\
\mathcal{Q}^{\lambda_{m}}(g)
\end{array}\right) \\
& =\left(\begin{array}{cccc}
\lambda_{1}^{q_{1}}\left(q_{1}+n\right) & \lambda_{1}^{q_{2}}\left(q_{2}+n\right) & \ldots & \lambda_{1}^{q_{m}}\left(q_{m}+n\right) \\
\lambda_{2}^{q_{1}}\left(q_{1}+n\right) & \lambda_{2}^{q_{2}}\left(q_{2}+n\right) & \ldots & \lambda_{2}^{q_{m}}\left(q_{m}+n\right) \\
\vdots & \vdots & \vdots & \vdots \\
\lambda_{m}^{q_{1}}\left(q_{1}+n\right) & \lambda_{m}^{q_{2}}\left(q_{2}+n\right) & \ldots & \lambda_{m}^{q_{m}}\left(q_{m}+n\right)
\end{array}\right) \\
& \quad \times\left(\begin{array}{c}
I_{1} \\
I_{2} \\
\vdots \\
I_{m}
\end{array}\right) .
\end{aligned}
$$

Once (13) is solved and the unknowns $I_{i}$ are found, the integration of $g$ follows as $\int_{\Omega} g(\mathbf{x}) d \mathbf{x}=\sum_{i=1}^{m} I_{i}$. The square matrix of coefficients in (13) depends on the degree of homogeneity of $g_{i}$ and the $m$ assumed values for $\lambda_{i}$, and the lefthand-side contains the operation of $n s p$-point quadratures $\mathcal{Q}^{\lambda_{i}}$ on the function $g$-no evaluation of $g_{i}$ is required. The quadrature construction algorithm in Sect. 2.2 is applied only once: when $\mathcal{Q}$ is created, $\mathcal{Q}^{\lambda_{i}}$ are obtained through the modification of the points of $\mathcal{Q}$.

\section{Example}

Consider the numerical integration of $g(x, y)=1+x^{2}+$ $y^{2}-2 y^{3}$ over the unit square $\Omega=[0,1]^{2} . g$ contains homogeneous functions $g_{1}=1, g_{2}=x^{2}+y^{2}$ and $g_{3}=-2 y^{3}$, with the degrees of homogeneity of 0,2 and 3 , respectively. For the sake of illustration, we select the degrees of homogeneity to be $q_{1}=0, q_{2}=1$, $q_{3}=2, q_{4}=3$, and assume $\lambda_{1}=1 / 4, \lambda_{2}=1 / 2$, $\lambda_{3}=3 / 4$ and $\lambda_{4}=1$. Using the above algorithm, we obtain: $I_{1}=1.000000000000000, I_{2}=0.000000000000003$, $I_{3}=0.666666666666662, I_{4}=-0.499999999999998$, and $I=\sum_{i=1}^{4} I_{i}=1.166666666666667$, which agrees with the exact integration of $g$ over the domain. Notice that $I_{2}=3 \times 10^{-15}$ is consistent with the fact that there is no term in $g$ with degree of homogeneity of 1 .

\section{Algorithm for the construction of quadratures on irregular convex polygons}

\subsection{Moment fitting equations}

A standard and well-known method for the construction of quadrature rules is the moment fitting equation [33-35] in which a quadrature is constructed for a class of basis functions over a fixed domain and a given weight function by solving the moment equation:

$$
\begin{aligned}
\left(\begin{array}{c}
\int_{\Omega} \omega(\mathbf{x}) \phi_{1}(\mathbf{x}) d \mathbf{x} \\
\int_{\Omega} \omega(\mathbf{x}) \phi_{2}(\mathbf{x}) d \mathbf{x} \\
\vdots \\
\int_{\Omega} \omega(\mathbf{x}) \phi_{m}(\mathbf{x}) d \mathbf{x}
\end{array}\right)= & \left(\begin{array}{cccc}
\phi_{1}\left(\mathbf{x}_{1}\right) & \phi_{1}\left(\mathbf{x}_{2}\right) & \ldots & \phi_{1}\left(\mathbf{x}_{n}\right) \\
\phi_{2}\left(\mathbf{x}_{1}\right) & \phi_{2}\left(\mathbf{x}_{2}\right) & \ldots & \phi_{2}\left(\mathbf{x}_{n}\right) \\
\vdots & \vdots & \vdots & \vdots \\
\phi_{m}\left(\mathbf{x}_{1}\right) & \phi_{m}\left(\mathbf{x}_{2}\right) & \ldots & \phi_{m}\left(\mathbf{x}_{n}\right)
\end{array}\right) \\
& \times\left(\begin{array}{c}
w_{1} \\
w_{2} \\
\vdots \\
w_{n}
\end{array}\right),
\end{aligned}
$$

where $\omega$ is the weight function, and $\Phi=\left\{\phi_{j}\right\}_{j=1}^{m}$ is the set of the basis functions defined over the domain of integration $\Omega$. The points of the quadrature and the corresponding weights, $\left\{\mathbf{x}_{i}, w_{i}\right\}_{i=1}^{n}$, are the unknowns that may be solved for algebraically or numerically so that the $m$ moment equations are satisfied. The resulting quadrature is exact (within the tolerance of the solution method) for the integration of the basis functions. The minimum number of integration points that can satisfy (14) is a classical problem of numerical analysis which has no solution yet [30]. Each integration point in $n$-dimensions, contributes $n+1$ unknowns (degrees of freedom): $n$ unknowns for its coordinate components and one for the weight. It can be seen that $m /(n+1)$ can serve as an estimate for the number of points required to integrate $m$ basis functions in $n$ dimensions.

Wandzura and Xiao [36] and Xiao and Gimbutas [30] applied Newton's least squares method to numerically solve (14) for the construction of high-order quadrature rules over the triangle, the square and the cube and then proposed the node elimination algorithm — one of the integration points was removed and (14) was solved again to get a quadrature with one fewer integration point. This procedure was continued until convergence of Newton's method could not be achieved anymore. Mousavi et al. [29] employed the node elimination algorithm to build symmetric quadratures for regular $n$-gons with $n=5,6,7$ and 8 . They also showed that the method is not restricted to regular domains-moderate degree close-to-minimal quadrature rules were produced over arbitrary convex and concave polygons. Node elimination algorithm was also used for the integration of discontinuous functions by replacing the weight function with a step function [32]. A nice feature of the node elimination algorithm is that the Newton iterations are only for the optimization of the quadrature rule and the output of each iteration is a solution of (14) by construction. One can stop the iterations as soon as the acquired quadrature is deemed suitable. In this paper, we fix the positions of the integration points 
Fig. 3 Algorithm for the construction of a quadrature for a Domain of integration; b homogeneous quadrature for the integration of basis functions; $\mathbf{c}$ locations of integration points (25 points); d final quadrature over the quadrilateral (21 points, relative norm of quadrature error $=4 \times 10^{-16}$ ); e locations of integration points are random combination of the polygonal vertices using barycentric coordinates (21 points, relative norm of quadrature error $=7 \times 10^{-16}$ ); $\mathbf{f}$ locations of integration points are selected as the grid points over a box containing the polygon ( 21 points, relative norm of quadrature error $=1 \times 10^{-16}$ ) an irregular convex polygon.

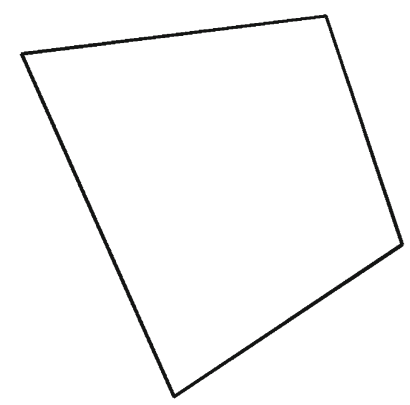

(a)

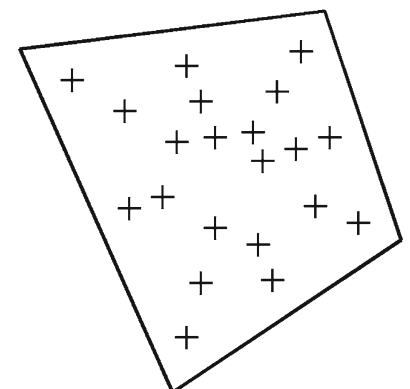

(d)

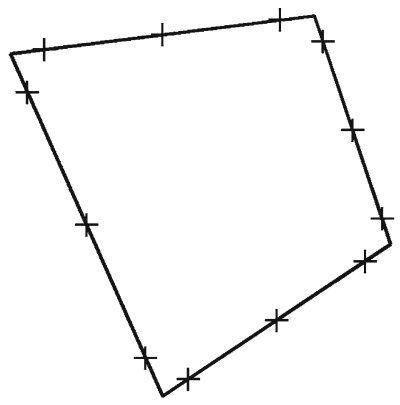

(b)

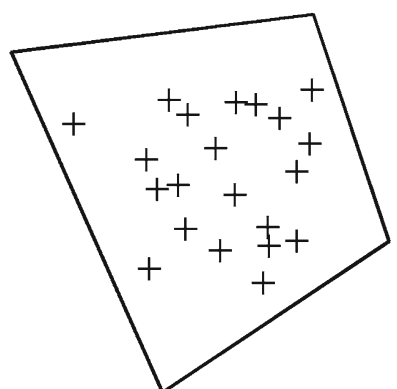

(e)

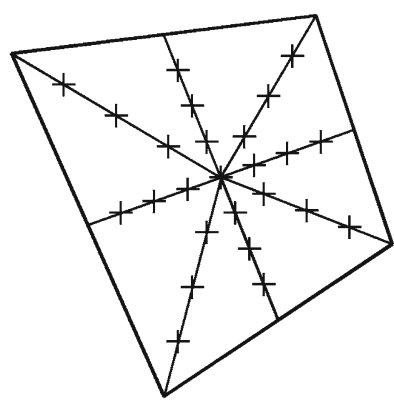

(c)

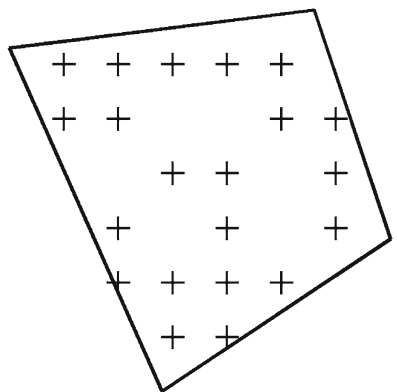

(f) over an irregular polygon and find the corresponding weights by solving (14). On knowing the locations of the integration points a priori, (14) turns into a linear system of $m$ moment equations and $n$ unknown weights. In general, the matrix of coefficients $A_{j i}=\phi_{j}\left(\mathbf{x}_{i}\right)$ can be non-square and we solve it in a least squares sense, that is, a solution with the minimum norm is sought. Solutions with some zeros are more desirable, because they imply fewer number of integration points.

Construction of a quadrature by moment fitting involves a one time calculation of the left-hand-side (lhs) of (14) that can be done algebraically (the integration boundaries are piecewise analytical curves) or numerically, using standard integration schemes such as Gauss quadrature rules. Divergence theorem can also be applied to convert the domain integrals into boundary integrals that can be carried out in a more straightforward manner [8,9]. In two dimensions, when the goal is to produce a quadrature for the integration of polynomials of order $d$ or lower, the set of basis functions $\Phi$ includes all bivariate monomials up to order $d, \mathbf{P}_{d}=\left\{x^{i} y^{j}, i, j \in\right.$ $\mathbb{Z}, i+j \leq d\}$. Note that $\mathbf{P}_{d}$ contains homogeneous functions and the method described in Sect. 2 can be applied to calculate $l h s$. Moreover, $\Phi$ can take on the form of any other set of basis functions that are linearly independent and cover the space of polynomials up to order $d$. For example, one may start with the monomials $\mathbf{P}_{d}$ and orthogonalize them for the given weight function over the integration region via a Gram-Schmidt procedure [29,30]. It bears mention that the integration of the orthogonal polynomials can be done using integration of homogeneous functions, of course with some extra steps.

\subsection{Description of the algorithm and examples}

In this section, we explain the algorithm for the construction of quadrature rules with polynomial-precision on irregular convex polygons. Consider the quadrilateral shown in Fig. 3a. We will construct a quintic quadrature using the moment fitting equations to illustrate the algorithm. The basis functions in (14) consist of bivariate monomials $x^{i} y^{j}, i+j \leq 5$ (21 basis functions), with the weight function $\omega$ set to unity. Therefore, we need a quadrature that can integrate polynomials of total order up to 5 to calculate the $l h s$. In one dimension, for example along the polygon edges, a 3-point Gauss quadrature rule is sufficient for the integration of polynomials up to order 5. Following the description provided in Sect. 2.2, a quadrature rule with 12 points is created with all the points lying along the edges (see Fig. 3b). This rule can integrate the basis functions using (6) and is then adopted to calculate the $l h s$ of (14). Having 21 equations to satisfy in (14), it is reasonable to expect at least 21 unknowns - there should be at least 21 integration points. For the locations of the integration points, we find the centroid of the polygon and connect it to the vertices and the middle of the edges and then pick 3 points over each of them (Fig. 3c). After solving the moment equations using a least squares fitting, 21 non-zero weights are obtained, corresponding to 21 integration points: this is a quadrature rule 
that can integrate polynomials of total order up to 5 over the polygon (see Fig. 3d). The positions of the integration points can be selected differently: for example, as a combination of the polygonal vertices using a barycentric coordinate with random coefficients (Fig. 3e). In Fig. 3f, a mesh-grid over the box containing the quadrilateral is constructed and the points that fall inside the domain are used as the integration points. The three quadratures shown in Fig. 3d-f, have 21 points and integrate polynomials of order 5 over the quadrangle. We calculate the relative norm of the quadrature error (called relative error from now on) as

$E_{\mathcal{Q}}^{r e l}=\frac{\|I(\Phi)-\mathcal{Q}(\Phi)\|}{\|I(\Phi)\|}$,

where, $\Phi$ is the set of numf basis functions and $I(\Phi)$ and $\mathcal{Q}(\Phi)$ are vectors of length numf with $I_{i}=\int_{\Omega} \phi_{i} d \mathbf{x}$ and $\mathcal{Q}_{i}=\mathcal{Q}\left(\phi_{i}\right)$. The relative error of these quadratures is of order $10^{-16}$. Some of the weights of the integration points are negative.

To show the versatility of the approach, we construct quadrature rules of orders 3,5 and 7 over irregular convex polygons with 4, 6, 8, 10,11 and 12 number of vertices (see Fig. 4). Figure $4 \mathrm{a}-\mathrm{f}$ presents quadratures of order 3 with the position of the integration points determined using diagonals and bisectors of the polygon, as shown in Fig. 3c. In two dimensions, there are 10 monomials in the space of polynomials up to order 3. Hence, 10 integration points are expected and realized, with the exception of the bi-unit square in Fig. 4a, which is a special case and fewer points are obtained. This can be explained in light of the symmetry of the square and the quadrature - the integral of many of the basis functions is identically equal to zero, which is satisfied by choosing a symmetric quadrature. The relative error of the quadrature in all these examples is of order $10^{-16}$. Figure $4 \mathrm{~g}-1$ shows quadrature rules of order 5 with random distribution of integration points. The relative error of the quadrature in this case is of order $10^{-13}$, which can be attributed to the poor distribution of the points. One can choose points that are more evenly distributed, e.g., by selecting a larger ensemble of random points. However, this increases the computation of the matrix of coefficients $A_{j i}$ in (14). We simply modified the random coefficients so that the integration points are attracted towards the polygon vertices and edges: the results are shown in Fig. $4 \mathrm{~m}-\mathrm{r}$ for order 5 quadratures. The relative norm of the quadrature error is reduced to the order of $10^{-15}$ as a result of better distribution of the integration points. This reveals the important effect of the locations of the integration points on the accuracy of the quadrature; at the same time, it indicates that one has the freedom of placing the points at certain locations of interest at the expense of losing some accuracy. Figure $4 s-x$ pertains to order 7 quadratures with mesh-grid distribution of integration points, with the relative norm of error of order $10^{-15}$. The lower accuracy of the quadrature is due to the ill-conditioning of the matrix of coefficients. The number of integration points for quadratures of order 5 and 7 are 21 and 36, respectively, which is equal to the number of basis functions in each case.

\section{Quadratures for discontinuous functions}

In many applications of numerical methods, the integrand may contain a discontinuity over the domain of integration. For example, such is the case in partition-of-unity methods like the extended finite element method for modeling cracks [15] and material interfaces [16]. Two different types of discontinuity exist in these applications: weak discontinuity, where the derivative of a continuous function is discontinuous, e.g., $|\mathbf{x}|$; and strong discontinuity, where the function itself is discontinuous, e.g., the generalized Heaviside function. A technique will be presented in the following sections to construct quadratures for handling strong and weak discontinuities. For some of the existing methods for integrating discontinuous functions, see Refs. [32,37-39].

\subsection{Strong discontinuities}

In this section, we construct efficient quadrature rules that can integrate discontinuous functions without partitioning the domain. For this purpose, similar to Ref. [32], we solve the moment equations (14) over the entire domain after replacing the weight function with a discontinuous function. The difference between our method and the technique presented in Ref. [32] is two-fold: (1) we evaluate the lhs of (14) using homogeneous quadratures presented in this paper, which results in fast and efficient evaluation of the integrals; and (2) we fix the locations of the integration points and solve a linear system of equations to obtain the corresponding weights. The number of integration points numx is proportional to the number of basis functions numf present in the moment equations (numx $\propto$ numf), and is not affected by the shape of the domain or the configuration of the discontinuity. A node elimination algorithm can still be applied to the final quadrature to reduce the number of integration points (in this case, one will have num $x \propto n u m f /(n+1)$ in $\left.\mathbb{R}^{n}\right)$, with the additional cost of optimization iterations. We will skip this step in the quadrature construction scheme presented here.

The algorithm for the construction of a discontinuous quadrature is similar to the one presented in Sect. 3.2 for polynomials, except that the weight function is set to the generalized Heaviside function and the $l h s$ of (14) is evaluated differently. We explain this step through an illustrative example. Consider an irregular convex polygon with the discontinuity shown in Fig. 5a. The generalized Heaviside function assumes a value of +1 above the discontinuity (in $\Omega^{+}$) and -1 below the discontinuity (in $\Omega^{-}$). We intend to 
Fig. 4 Examples of quadratures for irregular convex polygons with $4,6,8,10,11$ and 12 vertices. a-f Order 3 , uniform distribution of points as described in Sect. 3.2, relative norm of quadrature error of order $10^{-16} ; \mathbf{g}-\mathbf{l}$ order 5 , random distribution of points, relative norm of quadrature error of order $10^{-13} ; \mathbf{m}-\mathbf{r}$ order 5 , random distribution of points by manipulating the coefficients, relative norm of quadrature error of order $10^{-15} ; \mathbf{s}-\mathbf{x}$ order 7 , locations of integration points are selected from a mesh-grid over the polygon, relative norm of quadrature error of order $10^{-15}$

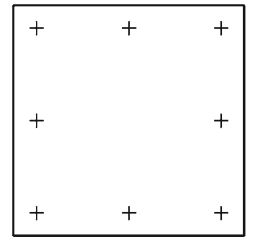

(a)

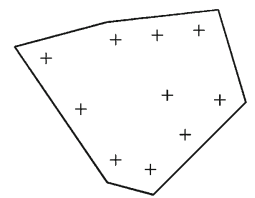

(b)

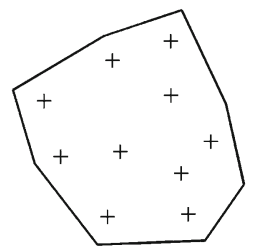

(c)

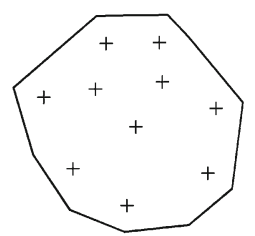

(d)

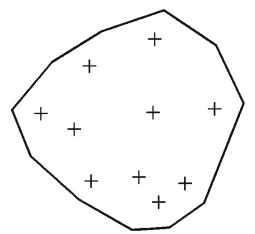

(e)

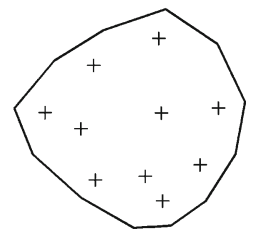

(f)

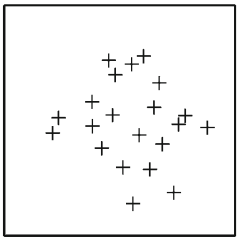

(g)

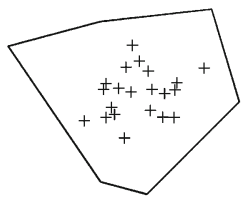

(h)

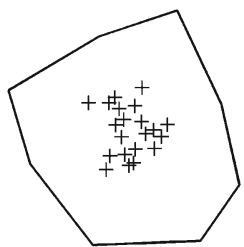

(i)

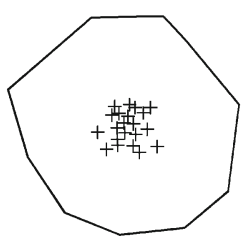

(j)

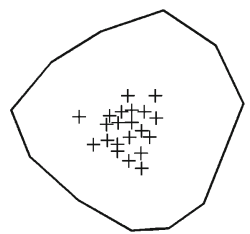

(k)

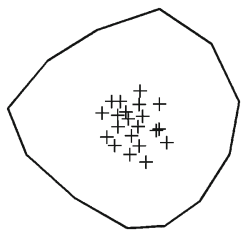

(I)

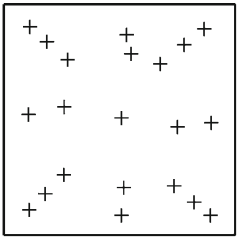

(m)

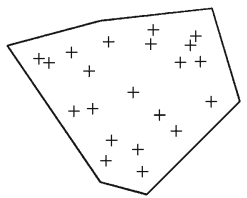

(n)

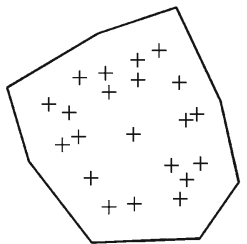

(o)

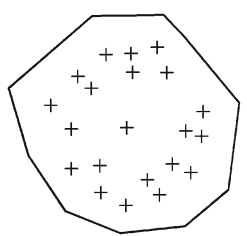

(p)

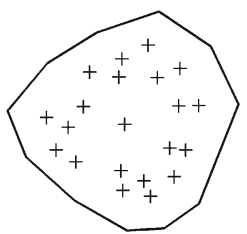

(q)

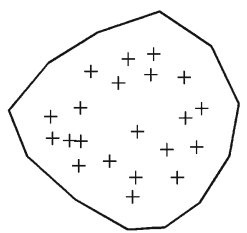

(r)

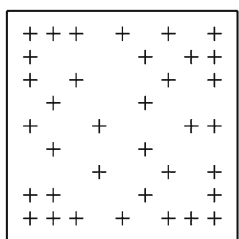

(s)

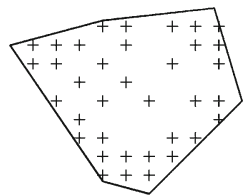

(t)

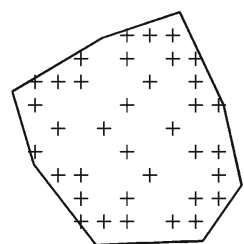

(u)

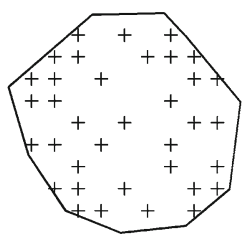

(v)

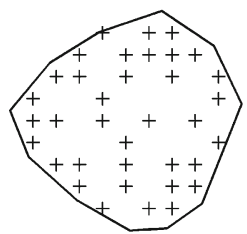

(w)

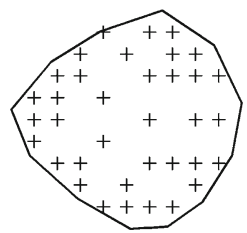

(x) create a discontinuous quadrature $\mathcal{Q}_{d}$ over $\Omega=\Omega^{+} \cup \Omega^{-}$ so that

$\mathcal{Q}_{d}(f(\mathbf{x}))=\int_{\Omega} f(\mathbf{x}) H(\mathbf{x}) d \mathbf{x}=\int_{\Omega^{+}} f(\mathbf{x}) d \mathbf{x}-\int_{\Omega^{-}} f(\mathbf{x}) d \mathbf{x}$,

where $f \in \mathbf{P}_{d}$ is any bivariate polynomial of total order up to $d$. Therefore, the basis functions include all bivariate monomials up to order $d$, i.e., the set of numf $=(d+1)$ $(d+2) / 2$ functions $\left\{x^{i} y^{j}, i+j \leq d\right\}$. To calculate the lhs of (14), we construct two homogeneous quadrature rules according to Sect. 2.2: $\mathcal{Q}_{\Omega^{+}}$over $\Omega^{+}$and $\mathcal{Q}_{\Omega^{-}}$over $\Omega^{-}$ (see Fig. 5b-c, respectively). The specific combination of these quadratures $\mathcal{Q}_{\Omega^{ \pm}}=\left\{\mathbf{x}_{i}, w_{i}, \mathbf{x}_{j},-w_{j}\right\}$ with $\left\{\mathbf{x}_{i}, w_{i}\right\}$ being the points and weights of $\mathcal{Q}_{\Omega^{+}}$and $\left\{\mathbf{x}_{j}, w_{j}\right\}$, the points and weights of $\mathcal{Q}_{\Omega^{-}}$, is used to evaluate lhs of (14) when the weight function $\omega(\mathbf{x})$ is set to $H(\mathbf{x})$. Once the $l h s$ is 
Fig. 5 Quadrature rules for the integration of discontinuous functions (third-order). Positions and weights of the integration points are given in Table 2. a Domain of integration with the vertices $\{(0,0),(3,1),(4,3),(3.5,4.5)$, $(-1,4)\}$ and discontinuity along the line segment $(-1.4,3.5)$ $-(4,1) ; \mathbf{b}$ and $\mathbf{c}$ homogeneous quadratures for the regions above and below the discontinuity; $\mathbf{d}$ discontinuous quadrature with random point locations; and e discontinuous quadrature with the points over a uniform grid

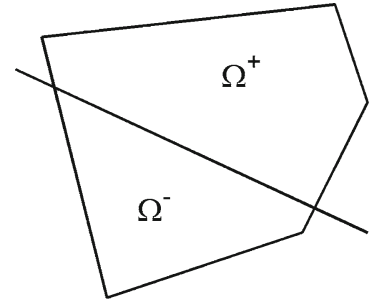

(a)

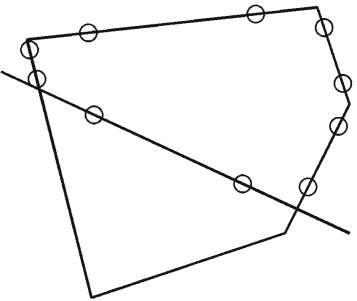

(b)

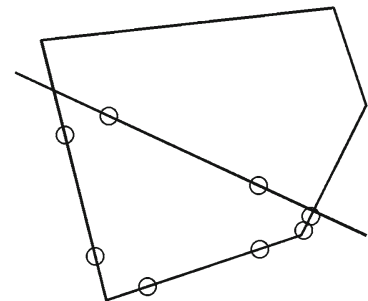

(c)

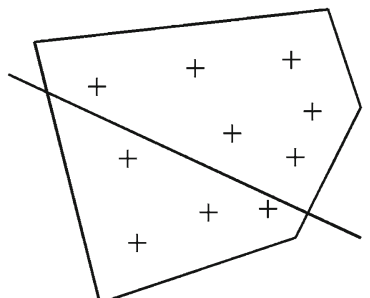

(d)

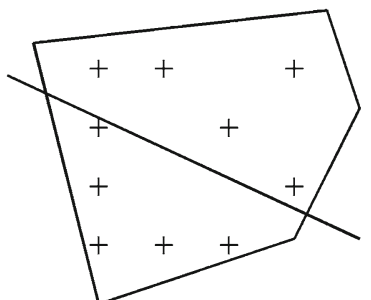

(e)

Table 2 Positions and weights of the third-order discontinuous quadrature of Fig. 5d-e

\begin{tabular}{|c|c|c|c|c|c|c|}
\hline \multirow[b]{2}{*}{$i$} & \multicolumn{3}{|l|}{ Random (Fig. 5d) } & \multicolumn{3}{|c|}{ Grid (Fig. 5e) } \\
\hline & $x$ & $y$ & Weight & $x$ & $y$ & Weight \\
\hline 1 & 2.030241295714621 & 2.597014923281813 & 5.259758278578383 & 0 & 0.9 & 1.587737294772927 \\
\hline 2 & 0.575502333815737 & 0.918565661655079 & 4.143633224938724 & 1 & 0.9 & 1.079974233075154 \\
\hline 3 & 2.576917760316822 & 1.445092921668437 & 6.512309005913230 & 2 & 0.9 & 1.382344556733680 \\
\hline 4 & 3.263901657120155 & 2.931503735932006 & 0.729577138635700 & 0 & 1.8 & -0.959879385332657 \\
\hline 5 & 2.936777960149114 & 3.724976205005987 & -2.218084949967891 & 3 & 1.8 & -1.139067959819840 \\
\hline 6 & -0.039262994659703 & 3.310174585512278 & -1.479457307747831 & 0 & 2.7 & 2.582736653755316 \\
\hline 7 & 1.670892704369278 & 1.387545264684462 & -6.176843962296525 & 2 & 2.7 & -0.209285340241211 \\
\hline 8 & 2.998733611229552 & 2.231920174438180 & -7.300223363309703 & 0 & 3.6 & -2.310728683746903 \\
\hline 9 & 1.464520359366653 & 3.598738697185424 & -4.218905860779358 & 1 & 3.6 & -1.802603037978114 \\
\hline 10 & 0.434344672882670 & 2.209435467495819 & 1.579104229133715 & 3 & 3.6 & -3.380361898119840 \\
\hline \multicolumn{2}{|c|}{ Integrand } & \multicolumn{5}{|c|}{ Relative error of integration } \\
\hline \multicolumn{2}{|c|}{$H(x, y)$} & \multicolumn{2}{|l|}{$2.8 \times 10^{-15}$} & \multicolumn{3}{|c|}{$1.8 \times 10^{-14}$} \\
\hline \multicolumn{2}{|c|}{$H(x, y) x$} & \multicolumn{2}{|l|}{$5.9 \times 10^{-16}$} & \multicolumn{3}{|c|}{$1.7 \times 10^{-15}$} \\
\hline \multicolumn{2}{|c|}{$H(x, y) y^{2}$} & \multicolumn{2}{|l|}{$6.8 \times 10^{-16}$} & \multicolumn{3}{|c|}{$6.8 \times 10^{-16}$} \\
\hline \multicolumn{2}{|c|}{$H(x, y) x y^{2}$} & \multicolumn{2}{|l|}{$1.7 \times 10^{-16}$} & \multicolumn{3}{|c|}{$6.8 \times 10^{-16}$} \\
\hline \multicolumn{2}{|c|}{$H(x, y)\left(x^{3}-x y+1\right)$} & \multicolumn{2}{|l|}{$4.0 \times 10^{-16}$} & \multicolumn{3}{|c|}{$1.6 \times 10^{-15}$} \\
\hline
\end{tabular}

calculated, the rest of the steps in the quadrature construction is similar to those for the integration of polynomials. See Fig. 5d-e for the final quadrature when the integration points are selected randomly and over a uniform grid, respectively. In this example, we construct a third-order quadrature rule with the points and weights given in Table 2. Also, we calculate the integration of bivariate monomials multiplied by the generalized Heaviside function and compare the results with the exact ones in Table 2-all the relative errors are of order $10^{-14}$ or smaller. Note that the first four functions in Table 2 belong to the set of basis functions used for the construction of the quadrature, and the last one is a nonhomogeneous polynomial times the generalized Heaviside function.

Since the homogeneous quadratures are only applicable to convex domains, it follows that $\Omega^{+}$and $\Omega^{-}$must also be convex so that $\mathcal{Q}_{\Omega^{+}}$and $\mathcal{Q}_{\Omega^{-}}$can be constructed. When the discontinuity has a single kink inside the integration region (see Fig. 6), for example, when a crack kinks inside a finite element, calculating the $l h s$ through the homogeneous quadratures is always possible, provided that the element $(\Omega)$ is convex. The reason is that depending on the configuration 


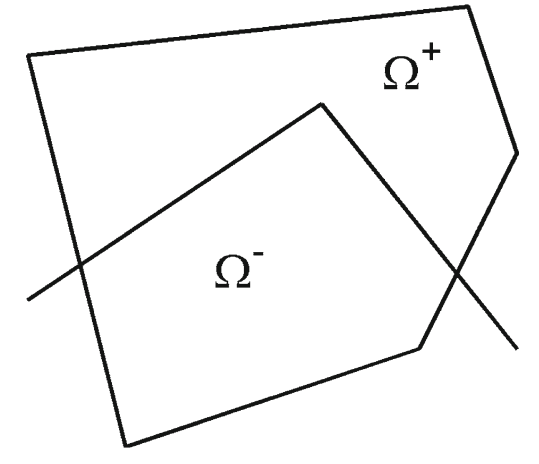

Fig. 6 Kink discontinuity

of the discontinuity, one of the subregions above or below the crack remains convex and one can write the integration over the concave subregion as the difference between the integration over the entire region and the convex subregion:

$$
\begin{aligned}
\int_{\Omega} f(\mathbf{x}) H(\mathbf{x}) d \mathbf{x} \\
=\left\{\begin{array}{l}
2 \int_{\Omega^{+}} f(\mathbf{x}) d \mathbf{x}-\int_{\Omega} f(\mathbf{x}) d \mathbf{x} \text { if } \Omega^{+} \text {is convex. } \\
\int_{\Omega} f(\mathbf{x}) d \mathbf{x}-2 \int_{\Omega^{-}} f(\mathbf{x}) d \mathbf{x} \text { if } \Omega^{-} \text {is convex. }
\end{array}\right.
\end{aligned}
$$

Therefore, the only added computation is to determine whether $\Omega^{-}$or $\Omega^{+}$is convex; then the lhs is calculated using two homogeneous quadratures, one over $\Omega$ and the other one over the convex subregion.

We show a few examples of quadrature rules for the integration of discontinuous functions in Fig. 7. Figure 7a-f shows fourth-order quadratures, each having 15 integration points with random distribution (there are 15 linearly independent monomials in $\mathbf{P}_{4}$ ). The discontinuity is along a straight line. The quadratures depicted in Fig. $7 \mathrm{~g}-1$ are sixth-order and for a kinked discontinuity with 28 integration points (random distribution). The relative norm of the quadrature error for all the quadratures of Fig. 7 is of order $10^{-14}$ or smaller.

\subsection{Weak discontinuities}

In case of a weak discontinuity, the integrand takes on the form of two different polynomials on either side of the interface. For this reason, two quadratures are constructed: one for each side (similar to the method in Ref. [39]). The resulting quadratures are exact and the total number of integration points over the cut element depends on the order of the quadrature, not on the shape of the element or the geometry of the interface, i.e., numx $\propto 2 \times$ numf for numf $=(d+1)(d+2) / 2$ when $d$ is the polynomial order of the quadrature. We present a simple illustrative example to show how quadratures over poly- gons can be used to integrate weakly discontinuous functions.

Consider the domain $\Omega=(-1,1) \times(-1,1)$ with the two subregions $\Omega_{1}$ and $\Omega_{2}$ shown in Fig. 8a. $\Omega_{2}$ is a circle with the radius $R$, centered at the origin, and $\Omega_{1}=\Omega \backslash \Omega_{2}$. The aim is to integrate a function $f(\mathbf{x})$ over $\Omega$ with $f=f_{1}$ over $\Omega_{1}$ and $f=f_{2}$ over $\Omega_{2}$. Figure $8 \mathrm{~b}-\mathrm{c}$, respectively, shows a surface and a contour plot of $f$ over the domain. To perform the integration, we divide the domain into numel $\times$ numel square divisions, similar to the finite element discretization. If an element is entirely inside $\Omega_{1}$ or $\Omega_{2}$, a Gauss quadrature rule is used to integrate the corresponding function. For the elements that are cut by the interface, the circular cut is approximated with numseg linear segments (see Fig. $8 \mathrm{~d}-\mathrm{e}$ ) and two quadratures are constructed for the two subregions. First, the quadrature over the convex subregion is constructed. The $l h s$ for the concave subregion is calculated by integrating over $\Omega$ and subtracting the contribution from the convex subregion, using the quadrature of the convex subregion. The exact integration can be performed algebraically. It should be noted that the error is caused by approximating a circle with linear segments (or equivalently, $\Omega_{2}$ is approximated with an $n$-gon), and the numerical integration over the partitions is exact within machine precision. The convergence curves of the relative error of integration are shown in Fig. $8 \mathrm{f}$ for numel $=\{10,20,40,80,160\}$ and numseg $=\{2,4,6\}$. Sample quadrature points for a $20 \times 20$ mesh with numseg $=4$ is shown in Fig. 8h-i when the subregions are partitioned into triangles and on using our integration scheme with random distribution of points, respectively. The rate of convergence in all cases is close to 2 , which is consistent with the relative error of approximating the area of a circle with $n$-gons, as $n$ is increased: $E_{r e l}=1-\frac{n}{2 \pi} \sin \frac{2 \pi}{n}$ (see analytical relative error in Fig. 8g). In Fig. 8g, we replace the circle with an $n$-gon and construct a quadrature over the $n$-gon. The filled squares in this figure pertain to the relative error in calculating the area of the circle using our quadratures (numerical relative error). To decrease the modeling error, the interface can be represented using higher-order curves as in Ref. [40].

\section{Extension to convex polyhedra}

In this paper, the main component of the quadrature construction scheme is the integration of the basis functions. We consider bivariate or trivariate monomials as the basis functions, and positions of the integration points can be selected randomly using barycentric coordinates. In two dimensions, we apply Theorem 1 to break the area-integrals into sum of lineintegrals over the boundary of the region that can be carried out efficiently. However, in three dimensions the situation is more complicated since now the boundary is comprised 
Fig. 7 Examples of discontinuous quadratures over irregular convex polygons with $4,6,8,10,11$ and 12 vertices. Locations of integration points are random with modified weights. a-f Fourth-order for a straight discontinuity, 15 integration points, relative norm of quadrature error of order $10^{-14} ; \mathbf{g}-\mathbf{l}$ sixth-order for a kinked discontinuity, 28 integration points, relative norm of quadrature error of order $10^{-14}$

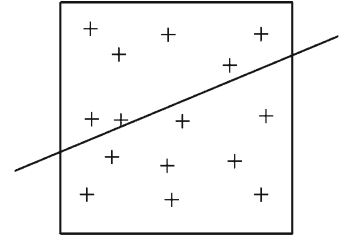

(a)

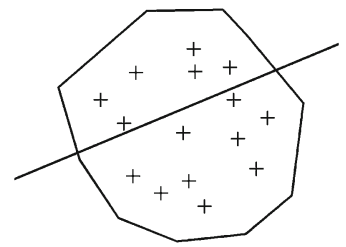

(d)

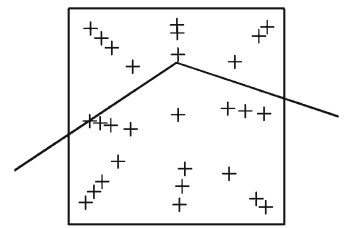

(g)

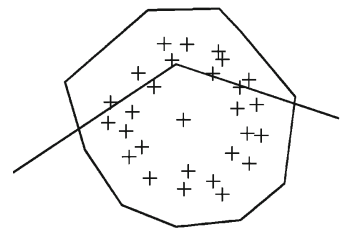

(j)

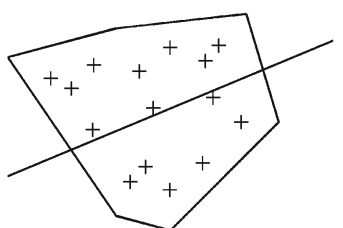

(b)

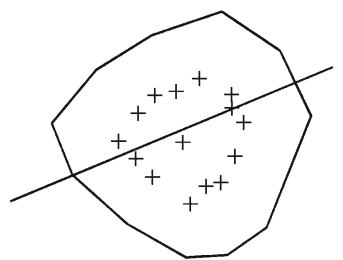

(e)

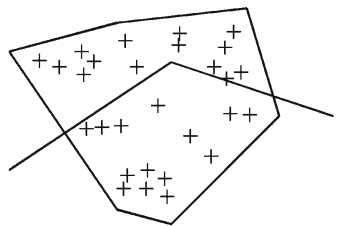

(h)

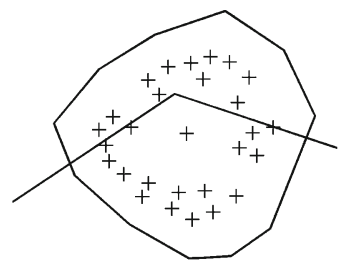

(k)

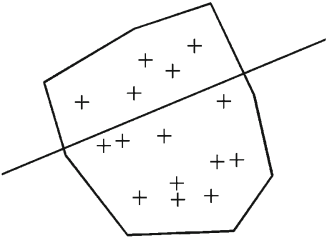

(c)

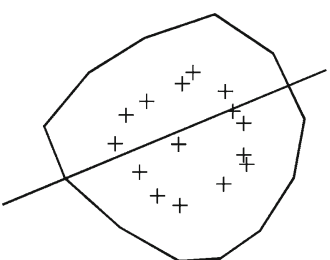

(f)

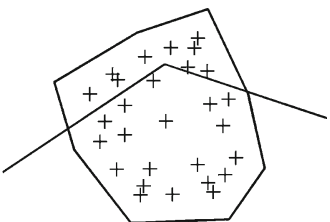

(i)

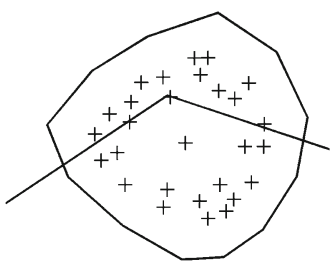

(l) of a few surfaces (we assume planar facets, i.e., convex polygons in three dimensions). Boundary integrations can be performed by subdividing them into triangles or by constructing quadratures over polygons, but neither of them is appealing due to the increased complexity. To overcome this difficulty, we use Theorem 2 and reduce the surface integrals over the faces of the polyhedron to one-dimensional integrals over the edges of the polyhedron (see (3)). Note that (3) contains the integration of the gradient of the basis functions over the faces of the domain. These integrations are circumvented by recursion without any added computational cost: the gradient of a monomial of total degree $p$ is another monomial of total degree $p-1$, which is also included in our basis functions. For example, for the monomials up to order $2,\left\{1, x, y, z, x^{2}, x y, x z, y^{2}, y z, z^{2}\right\}$, one starts the integration of the basis functions from the lowest order: $\phi=1$ with a zero gradient. Then the gradient of the linear terms $x, y, z$, is a constant and its integration over the boundary is available. The gradient of the second order terms are linear terms, and so on. With this approach, the surface integrals are suppressed and the volume integrals are performed using only line integrals. The following algorithm explains the method for the integration of trivariate monomials over an irregular convex polyhedron.

\section{Algorithm (Integration of trivariate monomials over an irregular convex polyhedron)}

Input: Highest order of monomials $d$, domain of integration $\Omega$ with the faces $\left\{\Omega_{i}\right\}_{i=1}^{m}$

Output: Integration of the monomials of total order up to $d$ over the domain $l h s$

1. Get the monomials $\Phi=\left\{x^{i} y^{j} z^{k}, i+j+k=p, p=\right.$ $0,1, \ldots, d ; i=p, p-1, \ldots, 0 ; j=p-i, p-i-$ $1, \ldots, 0\}$. Let $\phi_{j}$ be the $j$ th monomial. Number of basis functions is numf $=(d+1)(d+2)(d+3) / 6$.

2. Form the connectivity matrix $\mathbf{C}$ and the coefficient matrix $\mathbf{F}$ with the size numf $\times 3$ so that $\partial \phi_{j} / \partial x_{i}=F_{j i} \phi_{C_{j i}}$ (no summation implied) and $\left\{x_{i}\right\}_{i=1}^{3} \equiv\{x, y, z\}$. 
Fig. 8 Integration of weakly discontinuous functions.

a Domain of integration; b surface plot of the integrand; c contour plot of the integrand; d sample discretization of the domain and discontinuity; e dividing the cut element into two regions using a piecewise linear approximation of the discontinuity; $\mathbf{f}$ relative error of the integration; $\mathbf{g}$ relative error for calculating the area of the circle by approximating it by $n$-gons and using our quadratures; $\mathbf{h}$ integration points by partitioning the cut elements into triangles; and $\mathbf{i}$ integration points using our quadratures

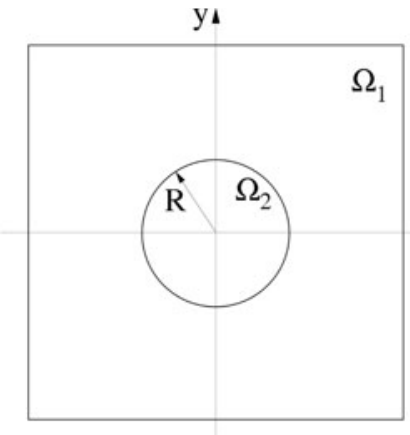

(a)

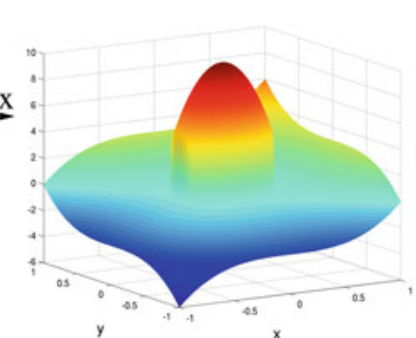

(b)

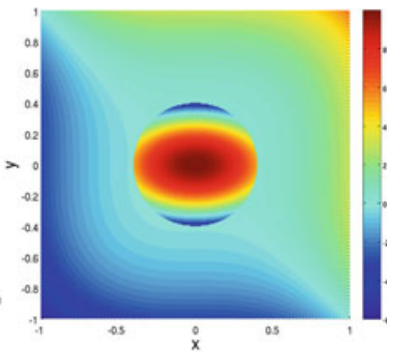

(c)

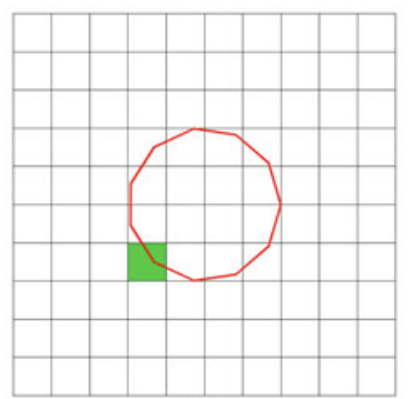

(d)

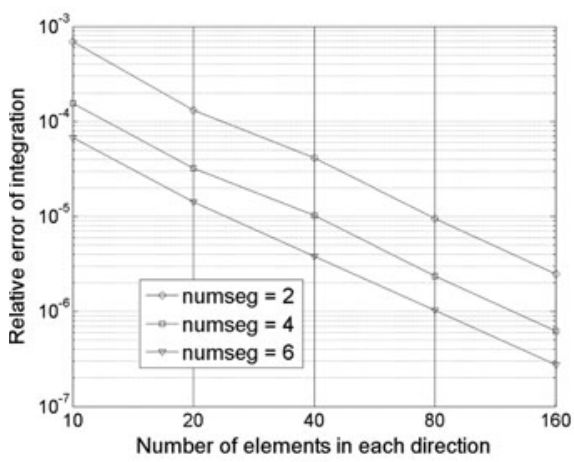

(f)

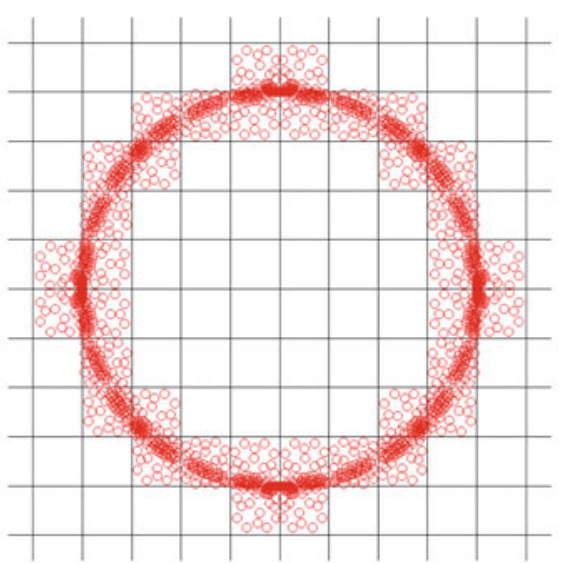

(h)

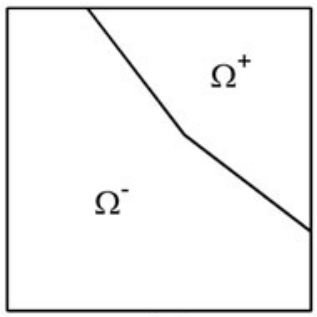

(e)

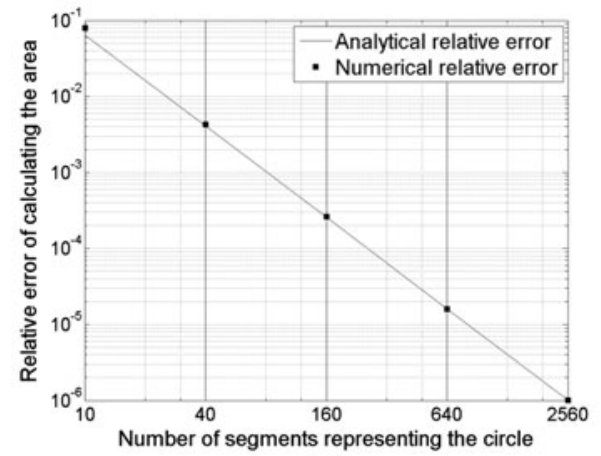

(g)

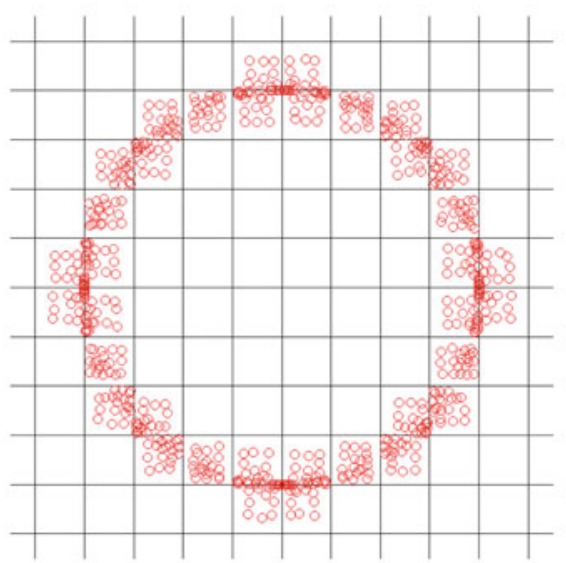

(i) 
3. Calculate the matrix $\mathbf{B}$ with the size numf $\times m$ using (3) so that $B_{j i}=\int_{\Omega_{i}} \phi_{j} d \mu$. Lower rows of $\mathbf{B}$, which pertain to higher orders of the monomials, are calculated using the higher rows that correspond to lower degrees of the monomials.

4. On computing the integral of all the monomials over the faces of the domain (B), use (2) to find $l h s$.

Remark Forming the matrices $\mathbf{C}$ and $\mathbf{F}$ is trivial and is only based on how the set of basis functions $\Phi$ is ordered. These matrices are only used to keep track of the gradients of the basis functions (express them in terms of the lower-order monomials). For example, the following shows the matrices $\mathbf{F}$ and $\mathbf{C}$ for the set of basis functions $\Phi=\left\{1, x, y, z, x^{2}, x y, x z, y^{2}, y z, z^{2}\right\}:$

$$
\mathbf{C}=\left(\begin{array}{lll}
1 & 1 & 1 \\
1 & 1 & 1 \\
1 & 1 & 1 \\
1 & 1 & 1 \\
2 & 1 & 1 \\
3 & 2 & 1 \\
4 & 1 & 2 \\
1 & 3 & 1 \\
1 & 4 & 3 \\
1 & 1 & 4
\end{array}\right) \text { and } \mathbf{F}=\left(\begin{array}{lll}
0 & 0 & 0 \\
1 & 0 & 0 \\
0 & 1 & 0 \\
0 & 0 & 1 \\
2 & 0 & 0 \\
1 & 1 & 0 \\
1 & 0 & 1 \\
0 & 2 & 0 \\
0 & 1 & 1 \\
0 & 0 & 2
\end{array}\right) .
$$

It is easy to verify that the equality $\partial \phi_{j} / \partial x_{i}=F_{j i} \phi_{C_{j i}}$ holds for $j=1,2, \ldots, 10$ and $i=1,2,3$. For example, for $j=8$ and $i=2$ one has: $\phi_{j}=y^{2}, F_{j i}=2, C_{j i}=3, \phi_{C_{j i}}=y$ and hence the equality $\partial y^{2} / \partial y=2 y$.

Remark The surface integration of the monomials in (3) is avoided through the following manipulation:

$$
\begin{aligned}
\int_{\Omega_{i}}\left\langle\nabla \phi_{j}, \mathbf{x}_{0}\right\rangle d \mu & =\int_{\Omega_{i}} \sum_{k=1}^{3} x_{0 k} \frac{\partial \phi_{j}}{\partial x_{k}} d \mu \\
& =\sum_{k=1}^{3} x_{0 k} \int_{\Omega_{i}} F_{j k} \phi_{C_{j k}} d \mu \\
& =\sum_{k=1}^{3} x_{0 k} F_{j k} B_{C_{j k} i},
\end{aligned}
$$

where $x_{0 k}$ is the $k$ th coordinate component of the point $\mathbf{x}_{0}$.

\subsection{Numerical examples}

\subsubsection{Irregular heptahedron: unit cube minus a tetrahedron}

As the first example, we construct a third-order quadrature (total order of the polynomials to be integrated exactly is 3 ) over the domain shown in Fig. 9. The integration region is a

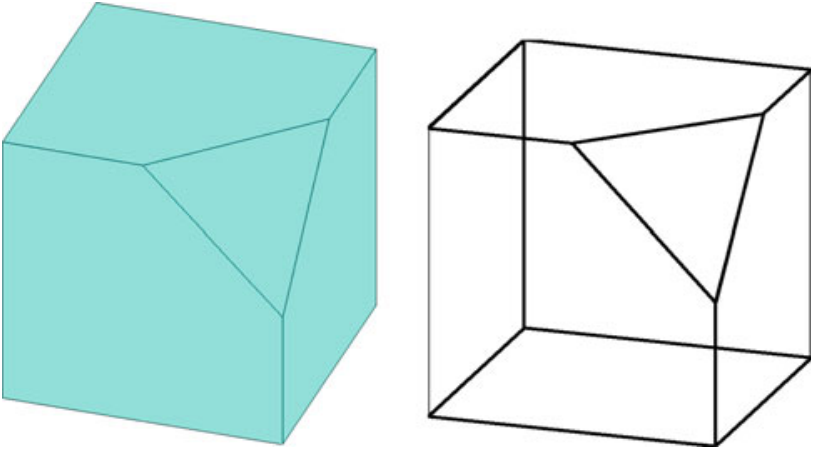

Fig. 9 Irregular heptahedron: unit cube minus a tetrahedron

Table 3 Coordinates of the vertices and connectivity of the faces of the irregular heptahedron

\begin{tabular}{llll}
\hline Vertices & $x$ & $y$ & $z$ \\
\hline 1 & 1 & 0 & 0 \\
2 & 1 & 1 & 0 \\
3 & 0 & 1 & 0 \\
4 & 0 & 0 & 0 \\
5 & 1 & $1 / 2$ & 1 \\
6 & 1 & 1 & 1 \\
7 & 1 & 1 & $1 / 2$ \\
8 & $1 / 2$ & 1 & 1 \\
9 & 0 & 0 & 1 \\
10 & 0 & & \\
\hline Faces & Connectivity & & \\
\hline 1 & $1,2,7,6,5$ & & \\
2 & $2,3,9,8,7$ & & \\
3 & $3,4,10,9$ & & \\
4 & $5,10,4,1$ & & \\
5 & $1,4,3,2$ & & \\
6 & $5,6,8,9,10$ & & \\
7 & $6,7,8$ & & \\
\hline
\end{tabular}

unit cube minus a tetrahedron constructed over one of the vertices of the cube. The region can be defined using 10 vertices and 7 faces with their coordinates and connectivity given in Table 3. There are 20 monomials in the basis function set of polynomials up to order 3 . These monomials are integrated with 576 basis function evaluations. Note that the basis functions are trivariate monomials and their evaluation at a given point is inexpensive. The final quadrature has 20 integration points as expected. See Table 4 for the positions and weights of the points. For the exact integration (to assess the accuracy of our quadrature), we integrate the monomials algebraically over the unit cube and then subtract the contribution of the tetrahedron. Quadrature rules for exact integration of polynomials over tetrahedrons are available in the literature (for example, see Refs. [41-43]). The relative error of the produced quadrature is of order $10^{-15}$. The relative error of 
Table 4 Positions and weights of a quadrature of total order 3 over the irregular heptahedron
The distribution of the points is random

\begin{tabular}{|c|c|c|c|c|}
\hline & $x$ & $y$ & $z$ & Weight \\
\hline 1 & 0.6319856330992721 & 0.3524980414890462 & 0.5412161514545703 & -0.9935165550012063 \\
\hline 2 & 0.6112764603706613 & 0.2842460438220646 & 0.3456663600269569 & 0.4033411913031856 \\
\hline 3 & 0.7476367049213167 & 0.3048071046880173 & 0.3881636404073822 & 0.3486049059653523 \\
\hline 4 & 0.3648365197758032 & 0.6166082077388840 & 0.2553757324215449 & 0.6661329163980730 \\
\hline 5 & 0.5074440285205465 & 0.7460607627741637 & 0.3985478210006686 & -0.0369030487247123 \\
\hline 6 & 0.4765105250092727 & 0.7079144951920009 & 0.3014705556484012 & -0.1503012511953231 \\
\hline 7 & 0.4104124440063532 & 0.4843159604043282 & 0.3126199335638323 & -0.5158977668411902 \\
\hline 8 & 0.2692967426831230 & 0.3394285421344097 & 0.3704014498867298 & 0.3070782226304006 \\
\hline 9 & 0.6741626711862849 & 0.2655279045109828 & 0.62476333345844989 & -0.0317604469387270 \\
\hline 10 & 0.5278539603101956 & 0.5783627395311751 & 0.5223108126435833 & -1.3252374753063219 \\
\hline 11 & 0.6571567227924915 & 0.5223598802072030 & 0.7874698497496101 & 0.2380062727700985 \\
\hline 12 & 0.7744535851182366 & 0.4747523895799468 & 0.6538825745568189 & 0.3340618048260526 \\
\hline 13 & 0.7698639943845597 & 0.6625050391811230 & 0.3944470991755144 & 0.0209383090819375 \\
\hline 14 & 0.6857658120815648 & 0.7421898806441541 & 0.4698496012443164 & 1.0239289693080991 \\
\hline 15 & 0.8413216125986441 & 0.7701327382165640 & 0.5201915416006866 & -0.2841310347465771 \\
\hline 16 & 0.5248245683630873 & 0.6774033818011266 & 0.7610592011515803 & -0.2510202750282448 \\
\hline 17 & 0.3089164830432281 & 0.6565782869258732 & 0.7789157096197711 & 0.5779875685280845 \\
\hline 18 & 0.4818905673003364 & 0.4508105701035444 & 0.6867311245003529 & 0.9238438135851143 \\
\hline 19 & 0.3906034486580987 & 0.4372177475524537 & 0.8125540888317655 & -0.8860899028736067 \\
\hline 20 & 0.4253557947192131 & 0.2923358620856172 & 0.7756170122266641 & 0.6101004489261785 \\
\hline \multicolumn{2}{|c|}{ Integrand } & \multicolumn{3}{|c|}{ Relative error of integration } \\
\hline \multicolumn{2}{|l|}{1} & \multicolumn{3}{|l|}{$5.6 \times 10^{-16}$} \\
\hline \multicolumn{2}{|l|}{$x$} & \multicolumn{3}{|l|}{$6.9 \times 10^{-16}$} \\
\hline \multicolumn{2}{|c|}{$x y^{2}$} & \multicolumn{3}{|l|}{$1.8 \times 10^{-16}$} \\
\hline \multicolumn{2}{|c|}{$z^{3}$} & \multicolumn{3}{|l|}{$5.8 \times 10^{-16}$} \\
\hline \multicolumn{2}{|c|}{$y^{3}-x y z+z^{2}+2$} & \multicolumn{3}{|l|}{$7.4 \times 10^{-16}$} \\
\hline
\end{tabular}

integration of a few trivariate monomials are also presented in Table 4: the quadrature is almost-exact for monomials with total order 3 or lower.

Due to absence of a quadrature for the integration over general convex polyhedra, one can subdivide it into tetrahedrons and then use quadratures over partitions for the numerical integration. In the case of the present example, the integration region can be divided into 16 tetrahedrons, and by using the 5-point cubic quadrature of Ref. [42], one obtains 80 integration points over the entire region. Furthermore, when partitioning is used, the number of integration points depends on the shape of the domain and connectivity of the faces in addition to the polynomial order of the quadrature, whereas in our algorithm, the number of integration points is proportional to the order of the quadrature, regardless of the shape of the domain.

\subsubsection{Irregular polyhedron with many faces}

To show the flexibility of the quadrature construction algorithm, we apply it to a more complicated domain. Consider

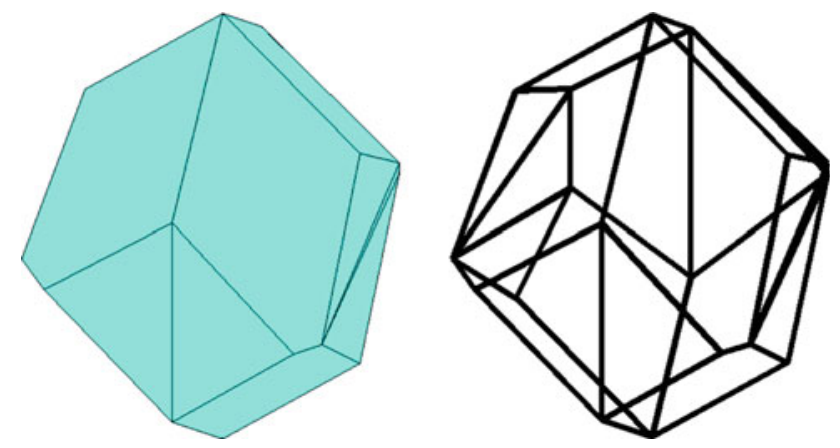

Fig. 10 Convex polyhedron with 18 vertices and 19 faces

the convex irregular polyhedron ${ }^{2}$ shown in Fig. 10 with 18 vertices and 19 faces given in Table 5. The algorithm for the construction of the polyhedron is described in Yip et al. [44]. We construct a cubic quadrature over the domain and present the weights and positions of the integration points in Table 6-the resulting quadrature has 20 integration points

\footnotetext{
$\overline{2 \text { An irregular }}$ polyhedron is one whose faces may be irregular
} polygons. 
Table 5 Coordinates of the vertices and connectivity of the faces of the convex polyhedron

\begin{tabular}{|c|c|c|c|}
\hline Vertices & $x$ & $y$ & $z$ \\
\hline 1 & 2.956100000000000 & 3.293900000000000 & 5.000000000000000 \\
\hline 2 & 2.998750000000000 & 5.000000000000000 & 3.251250000000000 \\
\hline 3 & 2.998750000000000 & 5.000000000000000 & 6.748750000000000 \\
\hline 4 & 3.043590000000000 & 6.793590000000000 & 5.000000000000000 \\
\hline 5 & 3.247500000000000 & 3.002500000000000 & 5.000000000000000 \\
\hline 6 & 5.000000000000000 & 5.000000000000000 & 1.250000000000000 \\
\hline 7 & 5.000000000000000 & 3.094740000000000 & 3.155260000000000 \\
\hline 8 & 5.000000000000000 & 3.094740000000000 & 6.844740000000000 \\
\hline 9 & 5.000000000000000 & 5.000000000000000 & 8.750000000000000 \\
\hline 10 & 3.530000000000000 & 7.280000000000000 & 5.000000000000000 \\
\hline 11 & 5.000000000000000 & 6.912500000000000 & 3.162500000000000 \\
\hline 12 & 5.000000000000000 & 6.912500000000000 & 6.837500000000000 \\
\hline 13 & 6.843040000000000 & 3.191740000000000 & 4.901300000000000 \\
\hline 14 & 6.843040000000000 & 3.191740000000000 & 5.098700000000000 \\
\hline 15 & 6.541670000000000 & 5.000000000000000 & 7.208330000000000 \\
\hline 16 & 6.276090000000000 & 6.593480000000000 & 5.880430000000000 \\
\hline 17 & 6.276090000000000 & 6.593480000000000 & 4.119570000000000 \\
\hline 18 & 6.541670000000000 & 5.000000000000000 & 2.791670000000000 \\
\hline Faces & Connectivity & & \\
\hline 1 & $2,1,3$ & & \\
\hline 2 & $5,1,2,6,7$ & & \\
\hline 3 & $5,8,9,3,1$ & & \\
\hline 4 & $10,4,2,6,11$ & & \\
\hline 5 & $10,4,3,9,12$ & & \\
\hline 6 & $5,7,13$ & & \\
\hline 7 & $9,15,14,8$ & & \\
\hline 8 & $9,15,16,12$ & & \\
\hline 9 & $10,12,16$ & & \\
\hline 10 & $6,7,13,18$ & & \\
\hline 11 & $6,18,17,11$ & & \\
\hline 12 & $15,16,17,18$ & & \\
\hline 13 & $2,3,4$ & & \\
\hline 14 & $5,13,14$ & & \\
\hline 15 & $5,14,8$ & & \\
\hline 16 & $10,16,17$ & & \\
\hline 17 & $10,17,11$ & & \\
\hline 18 & $15,18,13$ & & \\
\hline 19 & $15,13,14$ & & \\
\hline
\end{tabular}

and integrates all polynomials up to order 3 over the region (see the integration errors in Table 6). Similar to the previous example, for the calculation of the exact integrals, the domain is partitioned into tetrahedrons (32 tetrahedrons) and 5 -point tetrahedral quadratures are employed. The total number of basis function evaluation for the construction of the quadrature is 1152 .

\subsubsection{Three-dimensional discontinuous quadrature}

Next, we construct a discontinuous quadrature over a tetrahedral element with a kinked discontinuity. The coordinates of the vertices are $(0,0,3),(1,0,0),(1,1,2)$ and $(0,1,0)$ and the kinked discontinuity is contained in the two planes: $z=2.1$ and $y+z=2.6$ (see Fig. 11 for the geometry of the 
Table 6 Positions and weights of a quadrature of total order 3 over the convex polyhedron
The distribution of the points is random

\begin{tabular}{|c|c|c|c|c|}
\hline & $x$ & $y$ & $z$ & Weight \\
\hline 1 & 4.065709795565736 & 4.162155997653544 & 4.727895668889981 & -4.459294221326440 \\
\hline 2 & 3.945006242841623 & 4.296614254864239 & 5.032658948186411 & 22.87208005330276 \\
\hline 3 & 3.956317838457130 & 5.072225279458875 & 4.081156810615234 & -0.890007097566054 \\
\hline 4 & 4.020727362544046 & 5.152664082058308 & 5.790992552942226 & -10.61392242972703 \\
\hline 5 & 3.929908521745204 & 6.037653413585427 & 5.161937640050013 & 15.22165357649155 \\
\hline 6 & 4.984059196662368 & 5.146949262353924 & 3.188314234217299 & 34.12331154456583 \\
\hline 7 & 5.000688428115388 & 4.819017447794130 & 3.565541876877864 & -33.99782458590353 \\
\hline 8 & 5.075009194166556 & 4.144397175475000 & 3.845760521061140 & 13.35934747869531 \\
\hline 9 & 5.094651222398438 & 4.007924304779445 & 5.708146462338654 & -13.18999331236275 \\
\hline 10 & 4.867348506541593 & 3.834165799201663 & 6.297954735625012 & 5.291279053463872 \\
\hline 11 & 4.894822158847349 & 4.820980719998183 & 6.499507455597497 & -20.27676190196261 \\
\hline 12 & 4.890851876647310 & 4.819388016389012 & 6.879695758948051 & 26.41441472166087 \\
\hline 13 & 4.881218611500460 & 5.932051595186250 & 4.213149790015777 & -11.84276904057738 \\
\hline 14 & 4.848351922830217 & 5.963614996717538 & 5.844249251200039 & 2.458566427981979 \\
\hline 15 & 5.828300751817223 & 4.115615418801242 & 4.963750462040973 & 17.63578040648104 \\
\hline 16 & 5.924660436836890 & 5.112274393992814 & 6.296378681170026 & -0.582049204212033 \\
\hline 17 & 5.653195583059173 & 5.886988405049809 & 5.618342631081721 & 3.177444663755516 \\
\hline 18 & 5.558007746733869 & 5.604394796117942 & 4.702997087185103 & 8.896897888579491 \\
\hline 19 & 5.660217464475206 & 5.919982983444119 & 4.482252027916771 & 8.091914418585316 \\
\hline 20 & 5.715307400851893 & 4.972397189965120 & 4.082262791570251 & -10.58932553713920 \\
\hline \multicolumn{2}{|c|}{ Integrand } & \multicolumn{3}{|c|}{ Relative error of integration } \\
\hline \multicolumn{2}{|l|}{1} & \multicolumn{3}{|l|}{$7.3 \times 10^{-14}$} \\
\hline \multicolumn{2}{|c|}{$x$} & \multicolumn{3}{|l|}{$1.3 \times 10^{-15}$} \\
\hline \multicolumn{2}{|c|}{$x y^{2}$} & \multicolumn{3}{|l|}{$4.2 \times 10^{-16}$} \\
\hline \multicolumn{2}{|c|}{$z^{3}$} & \multicolumn{3}{|l|}{$7.0 \times 10^{-16}$} \\
\hline \multicolumn{2}{|c|}{$y^{3}-x y z+z^{2}+2$} & \multicolumn{3}{|l|}{$2.9 \times 10^{-15}$} \\
\hline
\end{tabular}

Fig. 11 A tetrahedron with a kinked discontinuity. a The geometry of the tetrahedron; and $\mathbf{b}$ and $\mathbf{c}$ configuration of the kinked discontinuity with a faceted plot and a wire plot, respectively

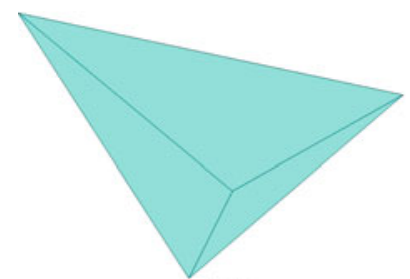

(a)

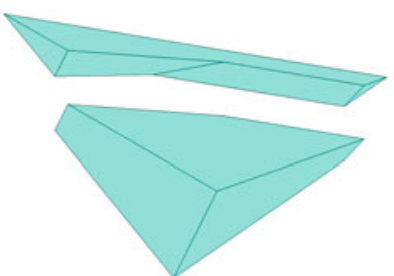

(b)

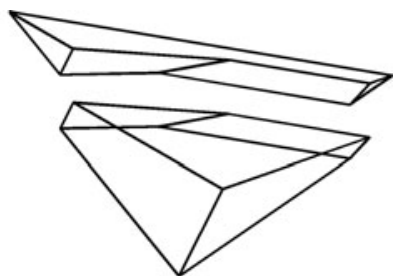

(c) domain and configuration of the discontinuity). To the best of the authors' knowledge no such quadrature is available in the literature due to the complexity of the domain of integration, and the only way to integrate the discontinuous functions over the domain is by splitting it into partitions over which quadratures are available. Knowing that the whole element (tetrahedron) and at least one of the divisions are convex, one can use our algorithm to integrate the basis functions. We use the generalized Heaviside function as the weight of the quadrature, which is equal to +1 in the top part and -1 in the bottom part. A cubic-order quadrature with 20 integration points is constructed over the prescribed cut element (see (14) and (16) for the construction and application of the quadrature). The positions and weights of the discontinuous quadrature and the error of integration for a few functions are given in Table 7 . The relative norm of the quadrature error is of order $10^{-15}$. The exact integration, for assessing the accuracy of the constructed quadrature, is performed 
Table 7 Positions and weights of a discontinuous quadrature of total order 3 over the tetrahedral region with a kinked discontinuity

The distribution of the points is random

\begin{tabular}{|c|c|c|c|c|}
\hline & $x$ & $y$ & $z$ & Weight \\
\hline 1 & 0.1079216435081310 & 0.1921795617491350 & 2.1869169638870836 & $-3.4967228111342404 \mathrm{e}-2$ \\
\hline 2 & 0.3669171402013682 & 0.5242212498412852 & 1.6003911521693723 & $-3.6559490812528794 \mathrm{e}-1$ \\
\hline 3 & 0.4197717668686998 & 0.5175403284264654 & 2.1029584801309684 & $7.1726907130123252 \mathrm{e}-2$ \\
\hline 4 & 0.2903258958952928 & 0.2030787521730345 & 2.0518317391067797 & $1.6667513346687882 \mathrm{e}-2$ \\
\hline 5 & 0.7734608718787533 & 0.7242319240942249 & 1.9766565558394811 & $-1.9032981859213351 \mathrm{e}-2$ \\
\hline 6 & 0.1794753580487591 & 0.3195668064538020 & 2.2814449955427860 & $7.5169545910981517 \mathrm{e}-2$ \\
\hline 7 & 0.7995222151169968 & 0.2898294265366389 & 0.9743594111317917 & $-6.0359297152038166 \mathrm{e}-2$ \\
\hline 8 & 0.6366396200268585 & 0.4345619228138845 & 1.7739511928746992 & $-1.0508713657398933 \mathrm{e}-1$ \\
\hline 9 & 0.5273418308141421 & 0.3909274012135061 & 0.6211105610358407 & $-4.6640612759658195 \mathrm{e}-2$ \\
\hline 10 & 0.7182437804258545 & 0.3279721455315767 & 0.3938212906355516 & $-2.3898179565953151 \mathrm{e}-2$ \\
\hline 11 & 0.6627864674694264 & 0.1482731620564133 & 0.6951081682731412 & $-6.3419482422352333 \mathrm{e}-2$ \\
\hline 12 & 0.8904666463249593 & 0.8134473164460133 & 1.5241767359161287 & $-2.4462222940832466 \mathrm{e}-2$ \\
\hline 13 & 0.6087797602119128 & 0.6157547781274360 & 1.4132657175141268 & $2.1828291064126790 \mathrm{e}-1$ \\
\hline 14 & 0.5591142497297681 & 0.8985413799029798 & 1.2882323421402608 & $-9.4525955981570708 \mathrm{e}-2$ \\
\hline 15 & 0.7575232763004042 & 0.9607222966033672 & 1.5029316407538882 & $3.5707485562175838 \mathrm{e}-2$ \\
\hline 16 & 0.2101307769911550 & 0.5184471122703831 & 0.9918617930864817 & $-2.2010185438599939 \mathrm{e}-2$ \\
\hline 17 & 0.1088855673897929 & 0.6993636220976017 & 0.5937580983275270 & $-1.0679725124058820 \mathrm{e}-3$ \\
\hline 18 & 0.1488562938657627 & 0.7881832864143193 & 0.2390370394327937 & $-5.2027165939133833 \mathrm{e}-2$ \\
\hline 19 & 0.4505413118541209 & 0.5918263700870281 & 0.4042741391488967 & $4.3965331968486124 \mathrm{e}-4$ \\
\hline 20 & 0.7094585777078288 & 0.6693188963782386 & 0.8921174548683573 & $-1.6027105689891447 \mathrm{e}-1$ \\
\hline \multicolumn{3}{|c|}{ Integrand } & \multicolumn{2}{|c|}{ Relative error of integration } \\
\hline \multicolumn{3}{|c|}{$H(x, y, z)$} & \multicolumn{2}{|l|}{$6.7 \times 10^{-16}$} \\
\hline \multicolumn{3}{|c|}{$H(x, y, z) x$} & \multicolumn{2}{|l|}{$3.2 \times 10^{-16}$} \\
\hline \multicolumn{3}{|c|}{$H(x, y, z) x y^{2}$} & \multicolumn{2}{|l|}{$1.1 \times 10^{-15}$} \\
\hline \multicolumn{3}{|c|}{$H(x, y, z) z^{3}$} & \multicolumn{2}{|l|}{$2.9 \times 10^{-15}$} \\
\hline \multicolumn{3}{|c|}{$H(x, y, z)\left(y^{3}-x y z+z^{2}+2\right)$} & \multicolumn{2}{|l|}{$1.3 \times 10^{-15}$} \\
\hline
\end{tabular}

using (17): the top division is prescribed in terms of the tetrahedron minus the bottom division which is partitioned into tetrahedrons for the sake of integration, and a 5-point quadrature over the tetrahedrons is used (13 tetrahedrons). The number of basis function evaluations for the quadrature construction is 576 .

\section{Concluding remarks}

We presented a technique for the integration of polynomials over irregular convex polygons and polyhedrons. While the position of the quadrature points were predetermined, moment equations were solved in order to obtain the corresponding weights. We chose the number of integration points to be greater than the number of equations, and then solved the moment equations in a least-squares sense to obtain the sparsest solution. With this technique, one has the freedom to select the integration points at desired locations. The number of integration points in the produced quadrature is proportional to the polynomial order of the quadrature, and is not affected by the geometry of the integration domain. In contrast to our approach, the number of integration points in the quadratures obtained through partitioning of the domain depends on the shape of the polytope in addition to the polynomial degree. For discontinuous functions and for subregions that are concave (e.g., due to a kinked discontinuity), the number of conformal subregions can grow and partitioning also becomes more challenging. Furthermore, adopting our quadrature scheme becomes favorable when the computation of the integrand is cost-intensive or when there is a need for the quadrature to be used repeatedly.

The added cost of our quadrature construction scheme is to calculate the integral of monomials over the domain, which was done using Lasserre's method-surface and volume integrals were reduced to line integrals using Euler's 
homogeneous function theorem, and then Gauss quadrature rule over the interval was employed. The resulting quadratures are almost-exact and can be used in the finite element method with irregular convex polygonal and polyhedral elements, as well as in the extended finite element method for weak discontinuities. In case of a strong discontinuity, we incorporated the generalized Heaviside function, which is a discontinuous function, as the weight in the moment equations - the resulting quadrature can integrate discontinuous functions (polynomials times the Heaviside function) over the cut polygonal/polyhedral element without partitioning it. The discontinuous quadrature can also be used over cut elements when there is a kink, provided that at least one of the regions remains convex. Several numerical examples were presented that demonstrated the application of the method for practical problems. Our algorithm permits accurate numerical integration of polynomials on irregular convex polygons and polyhedrons, and also alleviates the need to partition the elements for applications in the extended finite elements with cracks and material interfaces.

Acknowledgments The research support of the National Science Foundation through contract grants CMMI-0626481 and DMS-0811025 to the University of California at Davis is gratefully acknowledged. Helpful discussions with Matthias Köppe are also acknowledged.

Open Access This article is distributed under the terms of the Creative Commons Attribution Noncommercial License which permits any noncommercial use, distribution, and reproduction in any medium, provided the original author(s) and source are credited.

\section{References}

1. Wachspress EL (1975) A rational finite element basis. Academic Press, New York

2. Sukumar N, Tabarraei A (2004) Conforming polygonal finite elements. Int J Numer Methods Eng 61(12):2045-2066

3. Bishop JE (2009) Simulating the pervasive fracture of materials and structures using randomly closed packed Voronoi tessellations. Comput Mech 44(4):455-471

4. Talischi C, Paulino GH, Pereira A, Menezes IFM (2010) Polygonal finite elements for topology optimization: a unifying paradigm. Int J Numer Methods Eng 82(6):671-698

5. Wicke M, Botsch M, Gross M (2007) A finite element method on convex polyhedra. Comput Graph Forum 26(3):355-364

6. Martin S, Kaufmann P, Botsch M, Wicke M, Gross M (2008) Polyhedral finite elements using harmonic basis functions. Comput Graph Forum 27(5):1521-1529

7. Milbradt P, Pick T (2008) Polytope finite elements. Int J Numer Methods Eng 73:1811-1835

8. Rashid MM, Gullett PM (2000) On a finite element method with variable element topology. Comput Methods Appl Mech Eng 190(11-12):1509-1527

9. Rashid MM, Selimotic M (2006) A three-dimensional finite element method with arbitrary polyhedral elements. Int J Numer Methods Eng 67:226-252

10. Kaufmann P, Martin S, Botsch M, Gross M (2009) Flexible simulation of deformable models using discontinuous Galerkin
FEM. Graph Models 71(4):153-167. Special Issue of ACM SIGGRAPH/Eurographics Symposium on Computer Animation 2008

11. Voitovich TV, Vandewalle S (2007) Exact integration formulas for the finite volume element method on simplicial meshes. Numer Methods Partial Differ Equ 23(5):1059-1082

12. Brezzi F, Lipnikov K, Simoncini V (2005) A family of mimetic finite difference methods on polygonal and polyhedral meshes. Math Models Methods Appl Sci 15(10):1533-1551

13. da Veiga LB, Gyrya V, Lipnikov K, Manzini G (2009) Mimetic finite difference method for the Stokes problem on polygonal meshes. J Comput Phys 228:7215-7232

14. da Veiga LB, Lipnikov K, Manzini G (2010) Error analysis for a mimetic discretization of the steady Stokes problem on polyhedral meshes. SIAM J Numer Anal 48(4):1419-1443

15. Moës N, Dolbow J, Belytschko T (1999) A finite element method for crack growth without remeshing. Int J Numer Methods Eng 46(1):131-150

16. Sukumar N, Chopp DL, Moës N, Belytschko T (2001) Modeling holes and inclusions by level sets in the extended finite-element method. Comput Methods Appl Mech Eng 190(46-47):61836200

17. Lasserre JB (1998) Integration on a convex polytope. Proc Am Math Soc 126(8):2433-2441

18. Lasserre JB (1999) Integration and homogeneous functions. Proc Am Math Soc 127(3):813-818

19. Baldoni V, Berline N, De Loera JA, Köppe M, Vergne M (2010) How to integrate a polynomial over a simplex. Math Comput. doi:10.1090/S0025-5718-2010-02378-6

20. Hammer PC, Marlowe OJ, Stroud AH (1956) Numerical integration over simplexes and cones. Math Tables Other Aids Comput 10:130-137

21. Liu Y, Vinokur M (1998) Exact integrations of polynomials and symmetric quadrature formulas over arbitrary polyhedral grids. J Comput Phys 140:122-147

22. Lasserre JB, Avrachenkov KE (2001) The multi-dimensional version of $\int_{a}^{b} x^{p} d x$. Am Math Mon 108(2):151-154

23. Timmer HG, Stern JM (1980) Computation of global geometric properties of solid objects. Comput Aided Des 12(6):301-304

24. Cattani C, Paoluzzi A (1990) Boundary integration over linear polyhedra. Comput Aided Des 22(2):130-135

25. Bernardini $\mathrm{F}$ (1991) Integration of polynomials over $n$-dimensional polyhedra. Comput Aided Des 23(1):51-58

26. Mirtich B (1996) Fast and accurate computation of polyhedral mass properties. J Graph GPU Game Tools 1(2):31-50

27. Rathod HT, Govinda Rao HS (1997) Integration of polynomials over $n$-dimensional linear polyhedra. Comput Struct 65(6):829847

28. Dasgupta G (2003) Integration within polygonal finite elements. J Aerosp Eng 16(1):9-18

29. Mousavi SE, Xiao H, Sukumar N (2010) Generalized Gaussian quadrature rules on arbitrary polygons. Int J Numer Methods Eng 82(1):99-113

30. Xiao H, Gimbutas Z (2010) A numerical algorithm for the construction of efficient quadratures in two and higher dimensions. Comput Math Appl 59:663-676

31. Mousavi SE, Sukumar N (2010) Generalized Duffy transformation for integrating vertex singularities. Comput Mech 45(2-3): 127-140

32. Mousavi SE, Sukumar N (2010) Generalized Gaussian quadrature rules for discontinuities and crack singularities in the extended finite element method. Comput Methods Appl Mech Eng 199 (49-52):3237-3249

33. Lyness JN, Jespersen D (1975) Moderate degree symmetric quadrature rules for the triangle. J Inst Math Appl 15:19-32 
34. Lyness JN, Monegato G (1977) Quadrature rules for regions having regular hexagonal symmetry. SIAM J Numer Anal 14(2):283295

35. Dunavant DA (1985) High degree efficient symmetrical Gaussian quadrature rules for the triangle. Int $\mathrm{J}$ Numer Methods Eng 21:1129-1148

36. Wandzura S, Xiao H (2003) Symmetric quadrature rules on a triangle. Comput Math Appl 45:1829-1840

37. Ventura $G$ (2006) On the elimination of quadrature subcells for discontinuous functions in the eXtended finite-element method. Int $\mathrm{J}$ Numer Methods Eng 66:761-795

38. Holdych DJ, Noble DR, Secor RB (2008) Quadrature rules for triangular and tetrahedral elements with generalized functions. Int $\mathrm{J}$ Numer Methods Eng 73:1310-1327

39. Natarajan S, Mahapatra DR, Bordas SPA (2010) Integrating strong and weak discontinuities without integration subcells and example applications in an XFEM/GFEM framework. Int J Numer Methods Eng 83:269-294

40. Cheng KW, Fries TP (2010) Higher-order XFEM for curved strong and weak discontinuities. Int J Numer Methods Eng 82:564-590

41. Silvester $P$ (1970) Symmetric quadrature formulae for simplexes. Math Comput 24(109):95-100

42. Sunder KS, Cookson RA (1985) Integration points for triangles and tetrahedrons obtained from the Gaussian quadrature points for a line. Comput Struct 21(5):881-885

43. Keast $P$ (1986) Moderate-degree tetrahedral quadrature formulas. Comput Methods Appl Mech Eng 55:339-348

44. Yip M, Mohle J, Bolander JE (2005) Automated modeling of threedimensional structural components using irregular lattices. Comput-Aided Civil Infrastruct Eng 20:393-407 